\title{
Connectedness between crude oil and US equities: The impact of COVID-19 pandemic
}

\author{
Aktham Maghyereh \\ United Arab Emirates University \\ hussein abdoh ( $\nabla$ Hussein_abdoh@uaeu.ac.ae ) \\ United Arab Emirates University https://orcid.org/0000-0002-3352-3663
}

\section{Research}

Keywords: COVID-19 Pandemic, Oil, US Equity, Multifractaity, Cross-correlations, Connectedness

Posted Date: March 17th, 2021

DOl: https://doi.org/10.21203/rs.3.rs-301084/v1

License: (c) (i) This work is licensed under a Creative Commons Attribution 4.0 International License. Read Full License 


\title{
Connectedness between crude oil and US equities: The impact of COVID-19 pandemic
}

\begin{abstract}
In this paper, we exploit multifractal detrended cross-correlation analysis (MF-DCCA) to investigate the impact of COVID-19 pandemic on the cross-correlations between oil and US equity market (as represented by the S\&P 500 index). First, we examine the detrended moving average cross-correlation coefficient between oil and S\&P 500 returns before and during the COVID-19 pandemic. The correlation analysis shows that US stock markets became more correlated with oil during the pandemic in the long term. Second, we find that the pandemic has caused an increase in the long range cross correlations over the small fluctuations. Third, the MF-DCCA method shows that the pandemic caused an increase of multifractality in cross-correlations between the two markets. In sum, the pandemic caused a closer correlation between oil and US equity in the long range and a deeper dynamical connection between oil and US equity markets as indicated by the multifractality tests.
\end{abstract}

Keywords: COVID-19 Pandemic; Oil; US Equity; Multifractaity; Cross-correlations; Connectedness

JEL classifications: Q 43; G11; G01; G15 


\section{Introduction}

Since the start of COVID-19 virus in Wuhan in December 2019, the virus caused global health crises with 9,005,326 people infected and 468,712 deaths in 215 countries and territories as of June 21, 2020 (Worldometer Data Tracker (WDT), 2020). Governments imposed quarantine, isolation and social distancing which led to enormous economic repercussions. ${ }^{1}$ For example, The US unemployment rate increased $3.6 \%$ in January 2020 to 14.7 percent in April, the highest level since the Great Depression (Washington post report, 2020). ${ }^{2}$ Many researchers responded to the need to investigate the outcome of the pandemic on economy and stock markets. S\&P 500 index price declined almost 31\% from \$3,240.09 on December 30, 2019 to \$2,237.4 on March 23, 2020. According to Global Energy Review (2020), countries with full and partial lockdown witnessed on average a decline in energy demand by $25 \%$ and $18 \%$ per week, respectively. The West Texas Intermediate (WTI) oil experienced a fall by around 20\% two months following the start of the COVID-19 epidemic in Wuhan city.

Despite the quickly growing studies that examine the impact of COVID-19 on S\&P 500 (e.g., Gormsen and Koijen, 2020; Yilmazkuday, 2020; Baker et al., 2020; Cheng and Yen, 2020) and oil (e.g. Bakas and Triantafyllou, 2020; Sharif et al., 2020), our study is the first to examine the crosscorrelations and connectedness between oil and US equity during the pandemic. Also, this study is the first to examine the multifractality of oil and US equity returns during the pandemic. Specifically, we apply the multifractal detrended cross-correlation analysis (MF-DCCA) and the enhanced dynamic connectedness measure of Antonakakis et al., (2020) for analyzing the

\footnotetext{
${ }^{1}$ Well over 100 countries worldwide had instituted either a full or partial lockdown by the end of March 2020, Dunfor et al., (2020). https://www.bbc.com/news/world-52103747.

${ }^{2}$ The report can be accessed on this website: https://www.washingtonpost.com/business/2020/05/08/april-2020-jobsreport/)
} 
connectedness and cross-correlations between oil and US equity markets during the pandemic. The first measure allows examining the long-range correlation and the complexity of the relation between the two markets. Specifically, MF-DCCA can deduct long-range correlation taking into account the multifractality between financial time series. Multifractality in time series can be attributed to long-range temporal correlations or to the fat-tailed probability distributions of variations (Kantelhardt et al., 2002; and Zhou, 2008). The second measure is more flexible than Diebold and Y1lmaz (2014) approach because no rolling-window analysis is required, thus preserving the loss of observations from calculating the measure. Moreover, we do not need to arbitrarily set the rolling-window size.

This study focuses on the US market for two main reasons. First, crises in US market and their associated volatility and return outcome can be transmitted to other markets and sectors (see for example, Bekaert et al., 2014; Syriopoulos et al., 2015). Hence, our analysis of the influence of COVID-19 on the correlation between oil and US market sheds light on the correlation between oil and other stock markets. Second, the pandemic has caused the largest spread in terms of reported cases and deaths in United States, thereby studying the US markets provide unprecedented event for the correlation between oil and stock market returns.

Throughout our study, we compare between the period before the emergence of COVID-19 (January 1st, 2019 to December 31, 2019) and the period during the emergence of COVID-19 (January 1st, 2020 to October 30th, 2020) documenting the following findings. First, the detrended moving average cross-correlation between oil and S\&P 500 increases in the long term during the pandemic. Second, the small (rather than large) fluctuations of long range cross correlation increases during the pandemic indicating more consistency in the long correlations between oil and US equity market. Third, the multifractality in cross-correlations between the two markets has 
increased during the pandemic mainly due to the fat-tail distributions. Overall, the findings reveal that US stock markets are now more exposed to oil price fluctuation in the long term than before the pandemic.

In addition, using the dynamic connectedness measure of Antonakakis et al., (2020), we document the following two findings. First, during the early period of pandemic, the connectedness decreased steadily reaching a minimum value of approximately 7.5\% during February 2020. From that time, the trend reversed and started to increase upward reaching a maximum value of $25 \%$ during March of the same year. However, the connectedness measure decreased again reaching a lower level than observed before the pandemic. Second, we find that oil is a net transmitter of shocks to the forecast error variance of S\&P 500 during March, April and May, 2020 whereas S\&P 500 is a net transmitter to oil during the early period of the pandemic (January and February, 2020).

Our findings can offer practical implications to policy makers and investment practitioners. First, it is more complicated to predict the performance of S\&P 500 index based on oil during the pandemic due to high multifractality (complexity). Nevertheless, it is less difficult to predict the S\&P 500 performance during the long term horizon given the increase in long term crosscorrelations between oil and S\&P 500. Second, our findings confirm that modelling the dependence between oil and US equity may require using nonlinear (multifractal) econometric technique during the pandemic. Moreover, describing the dynamics of the cross-correlations during the pandemic require separating the short-term connectedness from the long term one. Third, investors who have allocations in stocks which are sensitive to oil price should not hold portfolio containing these stocks with fixed weight as the correlation and its multifractality fluctuate over time (i.e. short versus long terms). Finally, the emergence of S\&P 500 (oil) as a net transmitters during early (later) sample period of pandemic is a crucial finding from a dynamic (or 
time-varying) portfolio diversification standpoint because mitigating portfolio investment risk should focus on the asset that distributes risk to the other asset.

The rest of the paper is organized as follows. The next section includes a brief review of the related literature. Section 3 describes the methodology. Section 4 describes the data and descriptive statistics. Section 5 provides the empirical analysis. Finally, Section 6 concludes.

\section{Related Literature}

In this section, we summarize the main findings of studies relating to the impact of COVID19 on stock markets (mainly the S\&P 500 index) and oil, and the dependence between oil and stock markets using multifractal detrended cross-correlation analysis (MF-DCCA).

Onali (2020) examines the effect of COVID-19 cases and deaths on the conditional mean and volatility of the Dow Jones and S\&P500, finding that the pandemic affects the volatility but not the stock returns once changes in conditional volatility are controlled. Gormsen and Koijen (2020) examine how the COVID-19 revised dividend growth expectations in the US. They find that investors revised growth expectations down by $40 \%$ from the expected growth on the three-year horizon of around $18 \%$. Also, they argue that drop in the stock market is also likely to be driven by an increase in discount rates. Yilmazkuday (2020) finds that an increase in cumulative daily COVID-19 cases by one percent leads to a decrease in cumulative daily (monthly) S\&P 500 Index by about .01 (.03) percent, respectively, where the reduction is mainly observed in march 2020. Baker et al., (2020) find that the daily US stock market (as represented by the S\&P 500 index) experienced 18 market jumps out from 22 trading days, more than any period with the same number of trading, for the time period spanning from 24 February to 24 March 2020. Their findings are mainly caused by the COVID19 pandemic and its related government policy responses of restrictions on commercial (i.e. service) activity and social distancing. 
There also a few studies investigating the effect of COVID-19 pandemic on oil price volatility. Using wavelet coherence, Sharif et al., (2020) examine the connectedness between the COVID19, economic policy uncertainty, US stock market and crude oil prices. Their findings document that oil is the main source of systematic risk for the US stock market. In particular, oil price leads the US stock market over the 4 to 8-days frequency bands and the coherence between oil and US COVID-19 is high over the 16-days frequency band during the a sample period from January, 21, 2020 to March, 30, 2020. Albulescu (2020) studies the impact of COVID-19 new infection cases on oil prices, whilst controlling for the role of US economic policy uncertainty and financial volatility during the period from January 21- March 9, 2020. Using Autoregressive Distributed Lag Regression method, he finds that the new COVID-19 daily cases exert a marginal negative impact on the crude oil prices in the long run.

Bakas and Triantafyllou (2020) applied a multivariate VAR model to analyze the response of oil and gold commodity volatility to the uncertainty shock caused by the COVID-19 pandemic. In contrast to the above studies, they show that oil volatility response to the pandemic is negative. The main explanation resides on less uncertainty caused by the low aggregate demand and supply levels during the pandemic. ${ }^{3}$ This is against the findings of the positive response of US equity market volatility to the pandemic (as detailed earlier). Given the opposing effect of COVID-19 pandemic on oil and equity market, this study fills the gap by identifying the cross correlation between oil and US equity during the pandemic. So it is in this context that we use dynamic detrended correlation and multifractility tests to unveil the dynamic relation between these two markets during the pandemic.

\footnotetext{
${ }^{3}$ The pandemic uncertainty creates a higher elasticity of oil aggregate demand and supply and the equilibrium level is adjusted more by quantities rather than oil price. As such, oil price fluctuations (or oil volatility) decreases during the pandemic.
} 
Ferreira et al., apply the detrended cross-correlation to examine the relation between oil price and twenty different stock markets. Their results show an increase in correlations during the 2008 global financial crises. Ramirez et al., (2008) and Wang et al., (2011) were earlier studies that applied the detrended fluctuation analysis (DFA) in order to detect the long-range correlations in time as it has the advantage of dealing with short time size series. Kantelhardt et al., (2002) proposed a multifractal characterization into the DFA and hence created method called MF-DFA that can determine the multifractal scaling behavior in financial markets. Zhou (2008) extends the detrended cross-correlation analysis to multifractal method (MF-DCCA) to study the long-rang power-law cross-correlation of two non-stationary time series.

Ma et al., (2013) applied MF-DCCA to examine the cross-correlation relationship between crude oil and the stock markets of the BRIC countries finding a multifractal properties especially in the short term. Yang et al., (2016) compared between the MF-DCCA and vector auto-regression (VAR) model to investigate the cross-correlations and multifractality between oil and ten sectors of the Chinese market. They conclude that the VAR model cannot express dynamics of the crosscorrelations between oil and financial market. Accordingly, it is crucial that we use the MF-DCCA method to examine the complicated statistical correlations between oil and S\&P 500 during the COVID-19 pandemic.

\section{Methodology}

\subsection{MF-DCCA approach}

In this paper, we apply the MF-DCCA approach to analyze how COVID-19 changes the correlations between oil price and US equities. This procedure was first proposed by Zhou (2008) to investigate the correlation and multifractal characteristics of two series. The method combines 
between multifractal detrended fluctuation analysis (MF-DFA) suggested by Kantelhardt et al., (2002) and the detrended cross-correlation analysis (DCCA) method proposed by Podobnik and Stanley (2008). We describe the MF-DCCA in the following sections.

Assume that $x(i)$ and $y(i)$ are two time series which have the same length $N$, where $i=$ $1, \ldots, N .{ }^{4}$ We first obtain the cumulative deviation of the two time series as follows

$$
X(i)=\sum_{k=1}^{t}\left(x_{k-} \bar{x}\right), \quad Y(i)=\sum_{k=1}^{t}\left(y_{k-} \bar{y}\right) \quad i=1, \ldots, N
$$

where $\bar{x}$ and $\bar{y}$ are the means of the two time series, respectively. The cumulative deviation series $X(t)$ and $Y(t)$ are then divided into $N_{s} \equiv \operatorname{int}(N / s)$ nonoverlapping segments, each has the same length $s$. Since the length $N$ of the series may not be an integer and multiple of sub-interval length $s$, a short part segment will remain at the end of each series. In order to get all part of the series and obtain the $2 N_{S}$ sub-series altogether, we repeat the same dividing procedures starting from the opposite end. Then for each $2 N_{S}$ sub-series, the least squares method is applied in order to find the local trends $\tilde{X}^{v}(i)$ and $\tilde{Y}^{v}(i)$ with a polynomial of kth order fit as $\tilde{X}^{v}(i)=\alpha_{1} i^{k}+\alpha_{3} i^{k-1}+\cdots+$ $\alpha_{k} i+\alpha_{k+1}$ and $\tilde{Y}^{v}(i)=\beta_{1} i^{k}+\beta_{3} i^{k-1}+\cdots+\beta_{k} i+\beta_{k+1}$, where $i=1,2, \ldots, s ; k=1,2, \ldots ; v=$ $1,3 \ldots, 2 N_{s}$. Next, the detrended covariance $F^{v}(s, v)$ for each $v=1,3 \ldots, 2 N_{s}$ can be determined as

$$
F_{x y}^{v}(s, v)=\frac{1}{s} \sum_{i=1}^{s}\left|X_{(v-1) s+i}(i)-\tilde{X}^{v}(i)\right|\left|Y_{(v-1) s+i}(i)-\tilde{Y}^{v}(i)\right|
$$

And for each $v=N_{s}+1, N_{s}+2, \ldots, N_{s}$,

$$
F_{x y}^{v}(s, v)=\frac{1}{s} \sum_{i=1}^{s}\left|X_{N-\left(v-N_{S}\right) s+i}(i)-\tilde{X}^{v}(i)\right|\left|Y_{N-\left(v-N_{S}\right)}(i)-\tilde{Y}^{v}(i)\right|
$$

\footnotetext{
${ }^{4}$ Most notations and symbols in this section are obtained from Wang et al., (2020a,b) and Ji et al., (2020)
} 
Based on Eqs. (2) and (3), we can obtain the $q$ th-order fluctuation function $F_{x y}^{q}(s)$ by averaging the detrended covariances as

$$
F_{x y}^{q}(s)=\left\{\frac{1}{2 N_{s}} \sum_{v=1}^{2 N_{s}}\left[F^{2}(s, v)\right]^{\frac{q}{2}}\right\}^{\frac{1}{q}} \text { for } q \neq 0
$$

and

$$
F_{x y}^{0}(s)=\exp \left\{\frac{1}{2 N_{s}} \sum_{v=1}^{2 N_{s}}\left[F^{2}(s, v)\right]\right\} \text { for } q=0
$$

Now, we can analyze the scale behavior of the fluctuation function by observing the $\log -\log$ plots of $F_{x y}^{q}(s)$ versus time scale $s$ for each value of $q$. If there is a long-range cross-correlation between the two time series, the power-law relationship can be obtained as follows: $F_{x y}^{q}(s) \propto$ $s^{h_{x y(q)}}$, where $h_{x y(q)}$ is the generalized hurst exponent versus $q$. When the two series are identical, then MF-DCCA is reduces to the standard multifractal (MF-DFA). Furthermore, if $q>0$, then the $h_{x y(q)}$ reveals large fluctuation in the time series, and if $\mathrm{q}<0$, the $h_{x y(q)}$ reveals the small fluctuation. That is to say, the decreasing scaling exponent $h_{x y(q)}$ with the increasing of $q$ implies that long-range correlations in the series exhibit a multifractal behavior. However, when $h_{x y(q)}$ is independent of $q$, it indicates the long-range correlations in the series are monofractal. The degree of multifractality between two series can be measured by range of $h_{x y}(q)$; a higher value of $\Delta H_{x y(q)}=h_{x y\left(q_{\text {Minimum }}\right)}-h_{x y\left(q_{\text {Maximum }}\right)}$ implies a strong level of multifractal behavior.

In addition, the scaling exponent $h_{x y}(q=2)>0.5$ indicates that there is a cross-correlations between the two series are positive persistent. If $h_{x y}(q=2)<0.5$, the cross-correlations between the two series are negative persistent, meaning that the fluctuations between the two series of time series are opposite. Finally, $h_{x y}(q=2)=0.5$ means no correlations. 
In addition, the scaling exponent (Renyi exponent) $\tau_{x y}(q)$ is used to describe the nature of multifractal as follows:

$$
\tau_{x y}(q)=q h_{x y}(q)-1
$$

If the scaling exponent $\tau_{x y}(q)$ is not linear with q, the cross-correlation of the two series considered to be multifractal. Otherwise, it's considered to be a monofractal. To describe the singularity content of the time series, the multifractal singularity strength $\alpha_{x y}$ and multifractal singularity spectra $f_{x y}(\alpha)$ can be obtained by Legendre transform as

$$
\begin{gathered}
\alpha_{x y}=q h_{x y}(q)+q h_{x y}^{\prime}(q), \\
f_{x y}(\alpha)=q\left(\alpha_{x y}-h_{x y}(q)\right)+1
\end{gathered}
$$

A larger $\Delta \alpha_{x y}=\alpha_{x y_{\text {Minimum }}}-\alpha_{x y_{\text {Maximum }}}$ implies a stronger multifractality of cross-correlations, thereby showing a stronger cross-correlations between two time series and smaller $\Delta \alpha_{x y}$ indicates weaker multifractality. ${ }^{5}$

\subsection{Cross-correlation test}

To analyze the nonlinear cross-correlations between oil returns $(x)$ and US stock returns $(y)$, we apply cross correlation statistic $Q_{c c}(m)$ from Podobnik et al., (2009) by the following function:

$$
Q_{c c}(m)=N^{2} \sum_{i=1}^{m} \frac{X_{i}^{2}}{N-i}
$$

where the cross-correlation function $X_{i}$ is defined as

$$
X_{i}=\frac{\sum_{k=i+1}^{N}\left(x_{k} y_{k-i}\right)}{\sqrt{\left(\sum_{k=1}^{N} x_{k}^{2}\right)\left(\sum_{k=1}^{N} x_{k}^{2}\right)}}
$$

\footnotetext{
${ }^{5}$ We estimated the MF-DCCA model with MATLAB R19b using a code provided by Ji et al., (2020), which we modified for our purposes.
} 
This statistic test is approximately distributed as $\chi^{2}(m)$ with $m$ degrees of freedom. The null hypothesis of the test is that none of the first $m$ cross-correlation coefficient between two time return series is different from zero. Rejecting the null hypothesis (i.e., $Q_{c c}(m)>\chi^{2}(m)$ ) indicates that the cross-correlation between the two time return series is statistically significant.

To further examine the degree of cross-correlations, we use the detrended cross-correlation coefficient $\left(\rho_{D C C A}\right)$ introduced by Zebende (2011) and Kristoufek (2014) as follows;

$$
\rho_{D C C A}=\frac{\left[F_{x y}^{q}(s)\right]^{2}}{\left[F_{x}^{q}(s) F_{y}^{q}(s)\right]}, \quad \rho_{D C C A} \in[0,1]
$$

where $F_{x y}^{q}(s)$ denotes the detrended covariance's fluctuation function between $x$ and $y$ estimated from step 3 above. $F_{x}^{q}(s)$ and $F_{y}^{q}(s)$ represent detrended fluctuation function of $x$ and $y$ respectively. A value of $\rho_{D C C A}$ close to 1 indicates higher cross- correlation between two time series. A zero value of $\rho_{D C C A}$ implies no cross-correlation. A positive (negative) value of $\rho_{D C C A}$ indicates that the cross-correlation between two time series is persistent (anti-persistent).

\subsection{TVP-VAR connectedness approach}

To check if results stands another specification, we use the dynamic connectedness procedure based on TVP-VAR introduced recently by Antonakakis et al., (2020). This novel procedure allows both the VAR parameters and the variances to vary via a stochastic volatility Kalman Filter estimation instead of the original connectedness approach of Diebold and Y1lmaz (2009, 2012, 2014) which uses the rolling-window VAR. ${ }^{6}$ The TVP-VAR connectedness approach allows to

\footnotetext{
${ }^{6}$ Unlike the original Diebold-Yilmaz framework, the TVP-VAR connectedness approach (1) does not require to set the rolling window-size, thereby its results are not sensitive to window size selection, (2) there is no loss of observations as there is no need to arbitrarily set the rolling window-size, (3) it utilizes the entire sample, thereby there is no loss of observations, (4) it is not sensitive to outliers and/or skewness, and (5) it is adjusted immediately following structural changes (i.e., events), which in turn enables us to track connectedness shocks between the variables more clearly and in a timely manner (see e.g., Antonakakis et al., 2020; Andrada-Félix et al., 2020; Bouri et al., 2020).
} 
measure the dynamic and directional transmission mechanism between oil and US equities. Below is a brief overview of the TVP-VAR connectedness framework.

Let $\boldsymbol{y}_{t}$ and $\boldsymbol{y}_{t-1}$ be represented by $N \times 1$ and $N p \times 1$ dimensional vectors, respectively. The TVP-VAR model can be written as ${ }^{7}$

$$
\begin{array}{ll}
\boldsymbol{y}_{t}=\beta_{t} \boldsymbol{y}_{t-1}+\varepsilon_{t}, & \varepsilon_{t} \mid F_{t-1} \sim N\left(0, S_{t}\right) \\
\beta_{t}=\beta_{t-1}+v_{t}, & v_{t} \mid F_{t-1} \sim N\left(0, R_{t}\right)
\end{array}
$$

where $\beta_{t}$ is $N \times N$ dimensional time-varying coefficient matrix, $\varepsilon_{t}$ is $N \times 1$ vector of disturbances with an $N \times N$ time varying variance-covariance matrix, $S_{t}, v_{t}$ is $N^{2} \times 1$ dimensional vectors, and $R_{t}$ is $N^{2} \times N^{2}$ dimensional matrix.

Once the time-varying coefficients $\beta_{t}$ and $S_{t}$ from the above TVP-VAR model are estimated, the generalized impulse response functions (GIRF) and generalized forecast error variance decompositions (GFEVD) can be calculated as in the generalized connectedness procedure of Diebold and Y1lmaz (2014). The H-step ahead (scaled) generalized forecast error variance decomposition (GFEVD) of the $i^{\text {th }}$ variable due to shocks to the variable $j^{\text {th }}$ at time $t$ donated by $\theta_{i j, t}^{g}(H)$ can be calculated as:

$$
\theta_{i j, t}^{g}(H)=\frac{\sum_{t=1}^{H-1}\left(S_{i j . t}^{-1 / 2} A_{H . t} \sum_{t} \varepsilon_{i j, t}\right)^{2}}{\sum_{i=1}^{N} \sum_{t=1}^{H-1}\left(S_{i j . t}^{-1 / 2} A_{H . t} \sum_{t} \varepsilon_{i j, t}\right)}
$$

where $\sum$ is the variance matrix of the vector of errors $\varepsilon_{i j, t}$, and $\sum_{j=1}^{N} \theta_{i j, t}^{g}(H)=1, \sum_{i j=1}^{N} \theta_{i j, t}^{N}(H)=$ $N$. In order to get a unit sum of each row of the variance decomposition, we normalize each entry of the matrix by the row sum as

\footnotetext{
${ }^{7}$ Note that the text and notation in this section are quoted from Antonakakis et al., (2020).
} 


$$
\tilde{\theta}_{i j, t}^{g}(H)=\frac{\theta_{i j . t}^{g}(H)}{\sum_{j=1}^{N} \theta_{i j, t}^{g}(H)}
$$

In the equation above, note that the decomposition including own shocks in each variable sums to one, i.e. $\sum_{j=1}^{N} \theta_{i j, t}^{g}(H)=1$, and that the total decomposition over the two variables sums to $N$, i.e. $\sum_{j=1}^{N} \tilde{\theta}_{i j, t}^{g}(H)=N$. Based on $\tilde{\theta}_{i j . t}^{g}(H)$ we can compute the time-varying total connectedness index (TCI) as

$$
\operatorname{TCI}_{t}^{g}(H)=\frac{\sum_{\substack{i, j=1 \\ i \neq j}}^{N} \tilde{\theta}_{i j, t}^{g}(H)}{\sum_{i, j=1}^{N} \tilde{\theta}_{i j, t}^{g}(H)} \times 100=\frac{\sum_{\substack{i, j=1 \\ i \neq j}}^{N} \tilde{\theta}_{i j, t}^{g}(H)}{N} \times 100
$$

The TCI in (16) is used to measure the time-varying total connectedness index. The index measures the total information flow among the markets included in the system (i.e., oil and US equity markets). It is an off-diagonal addition of the proportions of the forecast error variance of $y_{i}$ to shocks to $y_{j}$, for $i \neq j$.

The directional connectedness variable $i$ receives from variable $j$ (FROM), can be calculated as

$$
S_{\circ i}^{g}(H)=\frac{\sum_{\substack{j=1 \\ i \neq j}}^{N} \tilde{\theta}_{i j}^{g}(H)}{\sum_{i, j=1}^{N} \tilde{\theta}_{i j}^{g}(H)} \times 100
$$

Similarly, the directional connectedness from the variable $i$ to variable $j$ (TO) is computed as

$$
S_{i^{\circ}}^{g}(H)=\frac{\sum_{\substack{j=1 \\ i \neq j}}^{N} \tilde{\theta}_{i j}^{g}(H)}{\sum_{i . j=1}^{N} \tilde{\theta}_{i j}^{g}(H)} \times 100
$$

Finally, by taking the difference between Eqs. (17) and (18), we can construct the net total directional connectedness (NET) as follows:

$$
S_{i}^{g}(H)=S_{i^{\circ}}^{g}(H)-S_{\circ}^{g}(H)
$$


The total net directional connectedness in (19) reveals which market (oil or S\&P 500) is prevailing in the information transmissions.

\section{Data}

The dataset consists of the daily S\&P 500 Composite Index and the West Texas Intermediate (WTI) crude oil. The empirical analysis includes 372 daily observations covering the period from the January 1st, 2019 to June 1st, 2020. Since our main focus in this study is on the effect of the COVID-19 pandemic, we include only the "normal" times that have not extreme disturbance events before the emergence of COVID-19. We use high frequency data (daily) in order to retain a high number of observations so to capture adequately the rapidity and intensity of the dynamic interactions among asset prices. The data were retrieved from Thomson Reuters DataStream. The daily continuously compounded returns for each time series, $r_{t}$ is computed as $r_{t}=\ln \left(\frac{p_{t}}{p_{t-1}}\right)$, where $p_{t}$ is the daily price at time $\mathrm{t}$.

To examine the impact of COVID-19 pandemic on the dynamic relationship between crude oil and US equities (as represented by the S\&P 500), we divide the time series into two sub-periods; one is the "normal" period before the emergence of COVID-19 (January 1st, 2019 to December 31, 2019) and the other period is during the emergence of COVID-19 (January 1st, 2020 to the end of the sample period). ${ }^{8}$ The time series of S\&P 500 Composite Index and the West Texas Intermediate (WTI) crude oil price during the two periods are shown in Figure 1. We also present the descriptive statistics of the rate of return for both S\&P 500 and oil in Table 1.

\section{Insert Table 1 here}

\section{Insert Figure 1 here}

\footnotetext{
8 The outbreak of COVID-19 pandemic was first identified in Wuhan, China, in 31 December 2019. Accordingly, we consider the COVID-19 period from 1st January 2020 to June 1st, 2020.
} 
The table shows that the standard deviation of US equity returns is remarkably higher during the pandemic $(0.0305)$ than before it $(0.0077)$. Also, the standard deviation of oil returns $(0.0924)$ is higher during the pandemic compared to the deviation before the pandemic $(0.0211)$. The increase in the volatility of US equity returns and oil was not matched by an increase in mean returns. In fact, on average, the return has decreased from .001 to -.0001 for US equity. Therefore, oil may not reduce the risk in equity investments since its standard deviation increases as well during the pandemic while its return remains on average at the same level. However, in order to judge the hedging properties of oil against equity investments, we need to examine the fractal cross-correlations as detailed in the following sections. Jarque-Bera statistics show that return distributions for US equity and oil returns series before and during the pandemic are not normally distributed as the null hypothesis of normal distribution is rejected at $1 \%$ significance level. The two time series can be characterized by fat tails because Kurtosis value is greater than 3 . The returns are non-stationary as indicated by augmented Dickey-Fuller tests. Given the non-normality and non-stationarity, using multifractal detrended cross-correlation analysis (MF-DCCA) method is well suited in this study. For example, Zhou (2008) used the method to study the correlation and multifractal characteristics of two non-stationary series.

As shown in Figure 1, it is clearly shown that S\&P index has a higher price than oil before the pandemic and its level even increased further during the first two months of year 2020 ( the start of the pandemic) while the oil decreased slowly. During March 2020, S\&P 500 index decreased sharply but start to increase during April. Specifically, the index reached the lowest level on 23 March and then rose by about $30 \%$ in the last trading day in April and kept increasing steadily to the end of our sample period. 


\section{Empirical results}

\subsection{Cross-correlation test}

Figure 2 represents the Log-Log cross-correlation statistics $Q_{c c}(m)$ between oil and S\&P 500 for the full sample and Figure 3 shows the correlation before and during the pandemic. The test statistic $Q_{c c}(m)$ is approximately $\chi^{2}(m)$ distributed with $m$ degrees of freedom. The critical value of the cross-correlation statistics $\chi^{2}(\mathrm{~m})$ is represented by the black line with at 5\% significant level. The red line denotes the cross-correlation statistic $Q_{c c}(m)$. This test statistics involves testing a null hypothesis that first $\mathrm{m}$ cross-correlation coefficient is different from zero. If the value of $Q_{c c}(m)$ is larger than the critical value of $\chi^{2}(m)$, then the cross-correlation between oil and S\&P 500 variables is significant.

\section{Insert Figure 2 here Insert Figure 3 here}

Figure 2 shows that the cross-correlation statistic test $Q_{c c}(m)$ is greater than the critical value over all chi-square distributions with degree of freedom $\log (m)$ from 0 to 3 . Hence, the null hypothesis of no cross-correlation is rejected, which implies that there is interactive correlation between S\&P 500 and oil returns. Figure 3, presents the $Q_{c c}(m)$ before and during the pandemic. It can be shown that during the pandemic, the cross-correlation is significantly positive and greater than the correlation before the pandemic at $5 \%$ level only when the log degrees of freedom of the chi-square distributions are greater than one.

In Figure 4, we present the detrended cross-correlation analysis $\left(\rho_{D C C A}\right)$ before and during the pandemic across different time scales $(s)$. Before the pandemic, there was a positive and significant short and long-range correlations whereas during the pandemic, only the long range correlations are significant. When the time scale is longer than 70 days, the cross-correlation is 
higher during the pandemic than before. In Table 2 we report the descriptive statistics of the correlation coefficient before and during COVID-19 pandemic. The mean and median correlation before the pandemic (with a value approximately equals to 0.739 and 0.74 , respectively) is remarkably higher than the correlation during the pandemic ( 0.482 and 0.391 , respectively). As indicated earlier, this is due to higher short-term correlation between oil and S\&P 500 before the pandemic. Overall, these results suggest that US stock markets are more exposed to oil return during the pandemic in the long term. Stock markets are typically characterized by nonlinear systems with multifractal and chaotic structures (Wen-Jong, Chin-Kun and Amritkar (2004); Zeng, Wang, and Xu (2017); Yao, Liu and Ju (2020)). Hence, in the following section, we focus on the multifractal detrended cross-correlation analysis (MF-DCCA) method to investigate the crosscorrelations between crude oil and US stock market.

\section{Insert Figure 4 here Insert Table 2 here}

\subsection{Multifractal detrended cross-correlation analysis (MF-DCCA)}

Based on the multifractal detrended cross-correlation (MF-DCCA) method, in Figure 5, we show the $\log -\log$ plots of the fluctuation function $F_{q}(s)$ versus time scale for different $q$ values $(q=-10,-8, \ldots, 8,10)$ between oil and US equities. The slope for each curve reflects the generalized cross-correlation Hurst exponent $h_{O i l, S \& P 500}(q)$. For negative $q$, the $h_{O i l, S \& P 500}(q)$ is associated with time scales of small fluctuations while the $h_{O i l, S \& P 500}(q)$ is associated with time scales of large fluctuations if $q$ is positive. If $h_{O i l, S \& P 500}(2)$ are greater than 0.5 , it then indicates that the cross-correlations of the time series pair (or the variability for each series) are positive persistent. If $h_{O i l, S \& P 500}(2)<0.5$, the cross-correlations of the time series pair are negative 
persistent, meaning that the fluctuations of time series move in opposite direction. If $h_{O i l, S \& P 500}(2)=0.5$, it means that there is no correlation.

\section{Insert Figure 5 here}

As can be seen from Figure 5, almost every line shows an increasing relation between fluctuation function and time scales $(s)$. Thus, we conclude that a power-low cross correlations exists between oil and US stock market. However, the relation is stronger when $q$ is negative indicating that small fluctuation dominates the large fluctuation in describing the long range cross correlation between oil and S\&P 500. However, during the pandemic, the power-low cross correlations became lower and even insignificant when the fluctuation is large (q greater than and zero). Over long time scales ( $\ln (s)$ is greater than 4), power-low cross correlations exists within the small fluctuations.

To characterize the multifractal nature of our two time series, in Figure 6 we present the crosscorrelation multifractal scaling exponent $\tau(q)$ (Renyi exponent) for $q$ from -10 to 10 . The scaling exponent values vary with $q$ from -10 to 10 and it shows a concave relation with $q$ more during the pandemic than before. Therefore, the pandemic caused a greater extent of multifractality in the cross-correlation. The same conclusion can be reached by observing Figure 7. The multifractal spectra of the cross-correlation exhibits a bell shape and the width of the shape is greater during the pandemic. Moreover, the multifractalities exist in the autocorrelations of oil and S\&P 500 series which are greater during the pandemic. As shown in Table 3, the spectra width $\left(\Delta \alpha=\alpha_{\max }-\alpha_{\min }\right)$ of the cross correlation during the pandemic $(0.573)$ is greater than before the pandemic (0.409) with a net difference equals to 0.164 . The spectra width for oil time series decreases from 0.527 to -0.2498 whereas the spectra width for S\&P 500 time series increases from 0.522 to 0.699 . 


\section{Insert Figure 6 here \\ Insert Figure 7 here \\ Insert Table 3 here}

Furthermore, to investigate the different changes of the cross-correlation exponent $h(q)$ with various values of $q$, we provide in Figure 8 the generalized Hurst exponents $h(q)$ spectrum of cross-correlations for the two periods with q varying from -10 to 10 .

\section{Insert Figure 8 here}

When $q=2$, the cross-correlation exponent, $h_{O i l, S \& P 500}(2)$, between the crude oil and the US stock market is larger than 0.5 during the pandemic while lower than 0.5 before the pandemic, suggesting that the two correlated series became more persistent or long-range dependence. With regard to Hurst exponent, $h(q)$, when $q=2$ for each time series, we note that the value is greater than 0.5 for oil series before the pandemic indicating that the series was positive and persistent. However, the series became less persistence and almost not auto correlated during the pandemic as the value became closer to zero. In contrast, the Hurst exponent of S\&P 500 is greater than 0.5 even during the pandemic

\subsection{The sources of multifractal and the impact of the COVID-19 pandemic}

As pointed out by et al., (2002) and Zhou (2008), the multifractality in time series can be attributed to long-range temporal correlations or to the fat-tailed probability distributions of variations. To study the main cause of multifractality for our time series pair, we construct shuffled and phase-randomized (surrogated) original series. ${ }^{9}$

\footnotetext{
${ }^{9}$ For detailed information on how to construct the shuffled and surrogated time series from original time series, see Ji, Zhang and Zhao (2020), Chen et al., (2016) and Wang et al., (2020, a and b).
} 
The cross- correlations generalized Hurst exponent $h(q)$ and multifractal spectrums the $\tau(q)$ of the original, shuffled, and surrogated series are shown in Figures $9 \& 10$ respectively.

The scaling exponent $h(q)$ of original, shuffled and surrogated series are presented in Figure 9. The findings reveal a significant difference of the relation between Hurst exponent and $q$ of original series and shuffled series, indicating that long-range correlations play a role in explaining the multifractality of cross-correlation between oil and US stock markets before the pandemic. However, during the pandemic, long-range correlations and fat-tail distributions play a role in explaining the multifractality. Similar conclusion can be reached if we compare the multifractal spectrums of original series to shuffled and surrogated series in Figure 10. Specifically, the width of singularity strength $\alpha$ for surrogated series is more different (greater) from the original series than the difference between shuffled series and the original series before and during the pandemic. Thus, fat tail distributions play a more significant role in explaining the multifractality of crosscorrelation between oil and US stock markets that does the long range correlations.

\subsection{Dynamic connectedness}

\section{Insert Figure 9 here}

Insert Figure 10 here

Figure 11 displays the total connectedness index between oil and S\&P 500 over different time periods. The value of the index varies over time showing a clear trend in certain sub-periods. For example, from June 2019, there was an increasing trend reaching a maximum of $20 \%$ in late December 2019. That is to say, $20 \%$ of the forecast error variance in oil and S\&P 500 can be explained by their cross connectedness. However, since the start of the pandemic, the connectedness decreased steadily reaching a minimum value of approximately $7.5 \%$ during February 2020. From that time, the trend reversed and started to increase upward reaching a maximum value of $25 \%$ during March of the same year. The time periods before and during the 
pandemic are characterized by heterogeneity in connectedness fluctuation where the period before the pandemic shows small fluctuations in connectedness values while the period during the pandemic shows large fluctuations. Nevertheless, the large fluctuations are limited only to the early time period of the pandemic. During the pandemic, we document that the connectedness between oil and S\&P 500 reveals large dynamic variability ranging between $1 \%$ and $25 \%$. Overall, the increase in connectedness during the pandemic is not long lasting as its level goes down below that level witnessed before the pandemic.

\section{Insert Figure 11 here}

Our connectedness framework allows us to examine the dynamic net-receiving or a nettransmitting position for each asset considered here. These are provided by Figure 12. Firstly, it should be noted that shocks transmission from oil and S\&P 500 are positive over all time periods indicating that these two markets are transmitter of shocks. Both assets show similar shocks transmission effects before the pandemic. In particular, their transmission effects increase steadily from October 2019 to January 2020. However, the Bi-directional connectedness to each asset behaves more heterogeneously over time during the pandemic. For example, innovations in oil price explain more than $15 \%$ of the forecast error variance of S\&P 500 market returns in certain periods during March 2020, whereas innovations in S\&P 500 explain around 5\% of the variance of oil market returns.

\section{Insert Figure 12 here}

In Figure 13, we study time-varying net directional and pairwise connectedness between oil and S\&P 500. That is, we find whether each asset is a net transmitter (positive value) or a net receiver of shocks (negative value) in each time point during the sample. Oil and S\&P 500 appear to frequently switch between a net transmitter and a net receiver roles before the pandemic. During 
the Pandemic, the roles last longer. Oil is a net transmitter of shocks to the forecast error variance of S\&P 500 during March, April and May, 2020, whereas S\&P 500 is a net transmitter to oil during the early period of the pandemic. The emergence of S\&P 500 (oil) as a net transmitters during early (later) sample period of pandemic is a crucial finding from a dynamic (or time-varying) portfolio diversification standpoint because mitigating portfolio investment risk should focus on the asset that distributes risk to the other asset.

\section{Insert Figure 13 here}

\section{Availability of data statement}

The data that support the findings of this study are available as a supplementary file.

\section{Conclusions}

In this paper, we examine how a fundamental commodity in industrial production, the oil, correlates with the return of US equity as represented by the S\&P 500 index during the ongoing COVID-19 health pandemic. By using multifractal detrended cross-correlation analysis (MFDCCA) we document the following findings. First, the cross-correlation between oil and S\&P 500 becomes more positive and persistent during the pandemic while the auto-correlation of oil becomes negative. Second, the cross correlation between oil and S\&P was positive and consistent across different time scales before the pandemic but its value varies now with the pandemic across time scales. Specifically, the correlation increases during the pandemic only in the long range. Finally, the strength of cross correlation of multifractality between oil and S\&P 500 increases during the pandemic indicating that the complexity between oil and US equity markets is now higher than before the pandemic. By using the dynamic connectedness measure of Antonakakis, Chatziantoniou, and Gabauer (2020), we document the following two findings. First, during the 
early period of pandemic, the connectedness decreased steadily reaching a minimum value of approximately $7.5 \%$ during February 2020. From that time, the trend reversed and started to increase upward reaching a maximum value of $25 \%$ during March of the same year. However, the connectedness measure decreased again reaching a lower level than observed before the pandemic by the end of our sample period, i.e. May 31, 2020. Second, we find that oil is a net transmitter of shocks to the forecast error variance of S\&P 500 during March, April and May, 2020 whereas S\&P 500 is a net transmitter to oil during the early period of the pandemic (January and February, 2020).

Our findings could be helpful for investors who consider the oil commodity in their investment portfolio. They should consider the differing impact of the pandemic on the correlation in short versus long time scales for their diversification benefits analysis. The findings also relate to firm managers because their firm value correlates with oil as it is an important input in most firms operation and production. Finally, predicting S\&P 500 performance could be harder during the pandemic due to the increase in multifractality, thereby causing a concern from using linear techniques to capture the interdependencies during the pandemic. Accordingly, future research concerning the COVID-19 may consider other econometric methods such as the (MF-DCCA).

\section{Compliance with Ethical Standards:}

Conflict of Interest: Both authors have no conflict of interest to declare.

Ethical approval: This article does not contain any studies with human participants or animals performed by any of the authors 


\section{References}

Albulescu, C. (2020). Do COVID-19 and crude oil prices drive the US economic policy uncertainty? arXiv preprint arXiv:2003.07591.

Alvarez-Ramirez, J., Alvarez, J., Rodriguez, E., \& Fernandez-Anaya, G. (2008). Time-varying Hurst exponent for US stock markets. Physica A: statistical mechanics and its applications, 387(24), 6159-6169.

Andrada-Félix, J., Fernandez-Perez, A. and Sosvilla-Rivero, S. (2020). Distant or close cousins: Connectedness between cryptocurrencies and traditional currencies volatilities. Journal of International Financial Markets, Institutions and Money, In press.

Antonakakis, N., Chatziantoniou, I., \& Gabauer, D. (2020). Refined measures of dynamic connectedness based on time-varying parameter vector autoregressions. Journal of Risk and Financial Management, 13(4), 84.

Bakas, D., \& Triantafyllou, A. (2020). Commodity price volatility and the economic uncertainty of pandemics. Economics Letters, 109283.

Baker, S. R., Bloom, N., Davis, S. J., Kost, K. J., Sammon, M. C., \& Viratyosin, T. (2020). The unprecedented stock market impact of COVID-19 (No. w26945). National Bureau of Economic Research.

Bekaert, G., Ehrmann, M., Fratzscher, M., \& Mehl, A. (2014). The global crisis and equity market contagion. The Journal of Finance, 69(6), 2597-2649.

Bouri, E., Cepni, O., Gabauer, D., \& Gupta, R. (2020). Return connectedness across asset classes around the COVID-19 outbreak. International Review of Financial Analysis. In press.

Chen, F., Tian, K., Ding, X., Miao, Y. and Lu, C. (2016). Finite-size effect and the components of multifractality in transport economics volatility based on multifractal detrending moving average method. Physica A: Statistical Mechanics and its Applications, 462, 1058-1066.

Cheng, H. P., \& Yen, K. C. (2020). Does COVID-19 Affect the Financial Market?. Available at SSRN 3578263.

Ciner, C. (2001). Energy shocks and financial markets: nonlinear linkages. Studies in Nonlinear Dynamics \& Econometrics, 5(3).

Diebold, F.X. and Yilmaz, K. (2009). Measuring financial asset return and volatility spillovers, with application to global equity markets. The Economic Journal, 119 (534), 158-171.

Diebold, F.X. and Yllmaz, K. (2012). Better to give than to receive: predictive directional measurement of volatility spillovers. International Journal of Forecasting, 28 (1), 57-66.

Diebold, F.X. and Y1lmaz, K. (2014). On the network topology of variance decompositions: measuring the connectedness of financial firms. Journal of Econometrics, 182 (1), 119-134. 
Ferreira, P., Pereira, É. J. D. A. L., da Silva, M. F., \& Pereira, H. B. (2019). Detrended correlation coefficients between oil and stock markets: The effect of the 2008 crisis. Physica A: Statistical Mechanics and its Applications, 517, 86-96.

Gormsen, N. J., \& Koijen, R. S. (2020). Coronavirus: Impact on stock prices and growth expectations. University of Chicago, Becker Friedman Institute for Economics Working Paper, (2020-22).

Ji, Q., Zhang, X. and Zhu, Y. (2020). Multifractal analysis of the impact of US-China trade friction on US and China soy futures markets. Physica A: Statistical Mechanics and its Applications, 542, in press.

Kantelhardt, J. W., Zschiegner, S. A., Koscielny-Bunde, E., Havlin, S., Bunde, A., \& Stanley, H. E. (2002). Multifractal detrended fluctuation analysis of nonstationary time series. Physica A: Statistical Mechanics and its Applications, 316(1-4), 87-114.

Kristoufek, L., (2014). Detrending moving-average cross-correlation coefficient: Measuring crosscorrelations between non-stationary series. Physica A: Statistical Mechanics and its Applications, 406, 169-175.

Ma, W. J., Hu, C. K., \& Amritkar, R. E. (2004). Stochastic dynamical model for stock-stock correlations. Physical Review E, 70(2), 026101.

Ma, F., Wei, Y., Huang, D., \& Zhao, L. (2013). Cross-correlations between West Texas Intermediate crude oil and the stock markets of the BRIC. Physica A: Statistical Mechanics and its Applications, 392(21), 5356-5368.

Maghyereh, A. and Al-Kandari, A. (2007). Oil prices and stock markets in GCC countries: new evidence from nonlinear cointegration analysis. Managerial Finance, 33 (7), 449-460

Onali, E. (2020). Covid-19 and stock market volatility. Available at SSRN 3571453.

Podobnik, B., Grosse, I., Horvatić, D., Ilic, S., Ivanov, P. C., \& Stanley, H. E. (2009). Quantifying cross-correlations using local and global detrending approaches. The European Physical Journal B, 71(2), 243.

Podobnik. B, and Stanley, H.E. (2008). Detrended cross-correlation analysis: a new method for analyzing two non-stationary time series. Physical Review Letters 100(8),084102.

Sharif, A., Aloui, C., \& Yarovaya, L. (2020). Covid-19 pandemic, oil prices, stock market and policy uncertainty nexus in the us economy: Fresh evidence from the wavelet-based approach. Oil Prices, Stock Market and Policy Uncertainty Nexus in the US Economy: Fresh Evidence from the Wavelet-Based Approach (April 13, 2020).

Syriopoulos, T., Makram, B., \& Boubaker, A. (2015). Stock market volatility spillovers and portfolio hedging: BRICS and the financial crisis. International Review of Financial Analysis, 39, 7-18. 
Wang, J., Shao, W. Kim, J. (2020a). Multifractal detrended cross-correlation analysis between respiratory diseases and haze in South Korea. Chaos, Solitons \& Fractals, 135, in press.

Wang, J., Shao, W. Kim, J. (2020b). Analysis of the impact of COVID-19 on the correlations between crude oil and agricultural futures. Chaos, Solitons \& Fractals, 136, in press.

Wang, Y., Wei, Y., \& Wu, C. (2011). Detrended fluctuation analysis on spot and futures markets of West Texas Intermediate crude oil. Physica A: Statistical Mechanics and its Applications, 390(5), 864-875.

Yang, L., Zhu, Y., Wang, Y., \& Wang, Y. (2016). Multifractal detrended cross-correlations between crude oil market and Chinese ten sector stock markets. Physica A: Statistical Mechanics and Its Applications, 462, 255-265.

Yao, C. Z., Liu, C., \& Ju, W. J. (2020). Multifractal analysis of the WTI crude oil market, US stock market and EPU. Physica A: Statistical Mechanics and its Applications, 124096.

Yilmazkuday, H. (2020). Covid-19 effects on the s\&p 500 index. Available at SSRN 3555433. Baur, D. G., \& Lucey, B. M. (2010). Is gold a hedge or a safe haven? An analysis of stocks, bonds and gold. Financial Review, 45(2), 217-229.

Zebende, G.F. (2011). DCCA Cross-correlation coefficient: quantifying level of cross-correlation. Physica A: Statistical Mechanics and its Applications, 390, 614-18.

Zeng, Y., Wang, J., \& Xu, K. (2017). Complexity and multifractal behaviors of multiscalecontinuum percolation financial system for Chinese stock markets. Physica A: Statistical Mechanics and its Applications, 471, 364-376.

Zhou, W.X. (2008). Multifractal detrended cross-correlation analysis for two nonstationary signals. Physical Review E, 77,166-211. 
Table 1: Descriptive statistics for the returns series

\begin{tabular}{|c|c|c|c|c|c|c|c|c|c|}
\hline & Mean & Median & Maximum & Minimum & Std. Dev. & Skewness & Kurtosis & Jarque-Bera & $\mathrm{ADF}$ \\
\hline \multicolumn{10}{|c|}{ Panel A: All sample (January 1st, 2019 to June 1st, 2020) } \\
\hline S\&P 500 & 0.0007 & 0.0009 & 0.0897 & -0.1277 & 0.0176 & -1.0047 & 18.3837 & $3680.6190 * * * *$ & $-5.9595 * * *$ \\
\hline Crude Oil-WTI & 0.0012 & 0.0000 & 0.3002 & -0.3883 & 0.0526 & -0.2058 & 21.2770 & $5110.7420 * * *$ & $-5.8485 * * *$ \\
\hline \multicolumn{10}{|c|}{ Panel B: Before the COVID-19 (January 1st, 2019 to December 31, 2019) } \\
\hline S\&P 500 & 0.0010 & 0.0009 & 0.0338 & -0.0302 & 0.0077 & -0.6363 & 6.4865 & $150.3806^{* * * *}$ & $-17.3727 * * *$ \\
\hline Crude Oil-WTI & 0.0011 & 0.0009 & 0.1369 & -0.0823 & 0.0211 & 0.5157 & 10.1480 & $569.3841 * * *$ & $-17.3314 * * *$ \\
\hline \multicolumn{10}{|c|}{ Panel C: During the COVID-19 (January 1st, 2020 to June 1st, 2020) } \\
\hline S\&P 500 & -0.0001 & 0.0007 & 0.0897 & -0.1277 & 0.0305 & -0.5786 & 7.0180 & $77.2191 * * *$ & $-15.6721^{* * *}$ \\
\hline Crude Oil-WTI & 0.0012 & 0.0000 & 0.3002 & -0.3883 & 0.0924 & -0.1483 & 7.7690 & $100.8366^{* * * *}$ & $-9.7628 * * *$ \\
\hline
\end{tabular}

Note: ADF is the Augmented Dickey-Fuller unit root test.. ***Indicates statistical significance at $1 \%$ level.

Table 2: Descriptive statistics for the correlation coefficients $\left(\rho_{D C C A}\right)$ in both sub-periods

\begin{tabular}{cccccccc}
\hline & Mean & Median & Maximum & Minimum & Std. Dev. & Skewness & Kurtosis \\
\hline Before COVID-19 & 0.7386 & 0.7402 & 0.7947 & 0.6690 & 0.0349 & -0.1347 & 2.0161 \\
During COVID-19 & 0.4816 & 0.3907 & 0.7936 & 0.2067 & 0.2256 & 0.3383 & 1.3899 \\
\hline
\end{tabular}

Table. 3: Multifractal spectra widths $\Delta \alpha$

\begin{tabular}{lccc}
\hline & Cross & Crude Oil-WTI & S\&P 500 \\
\hline Before COVID-19 & 0.4090 & 0.5270 & 0.5216 \\
During COVID-19 & 0.5727 & -0.2498 & 0.6990 \\
Net & 0.1637 & -0.7768 & 0.1774 \\
\hline
\end{tabular}




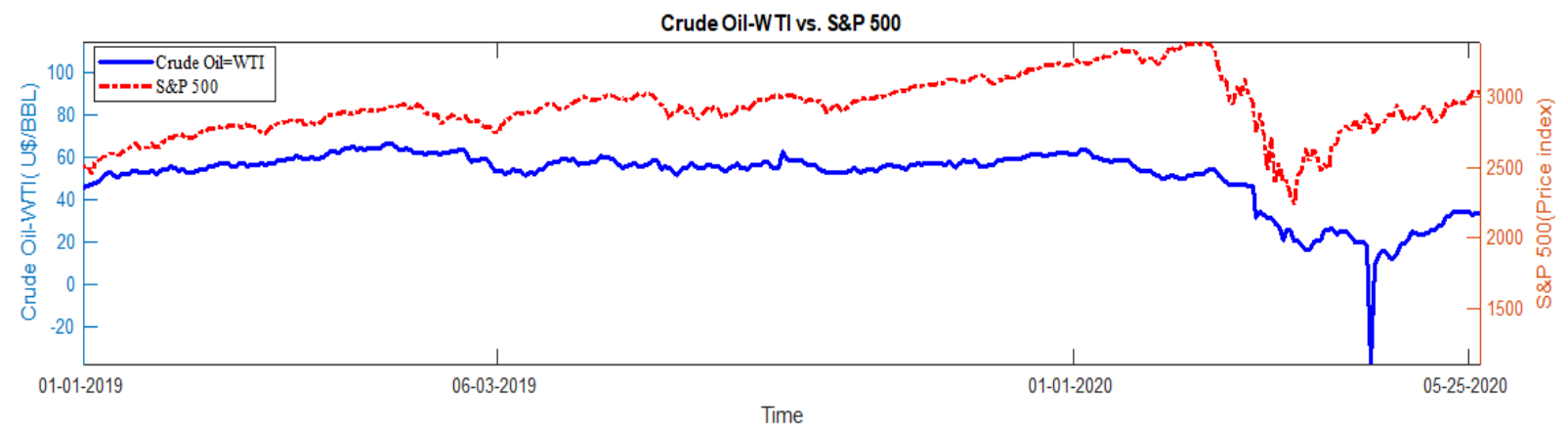

Figure 1: Time evolutions for the time series

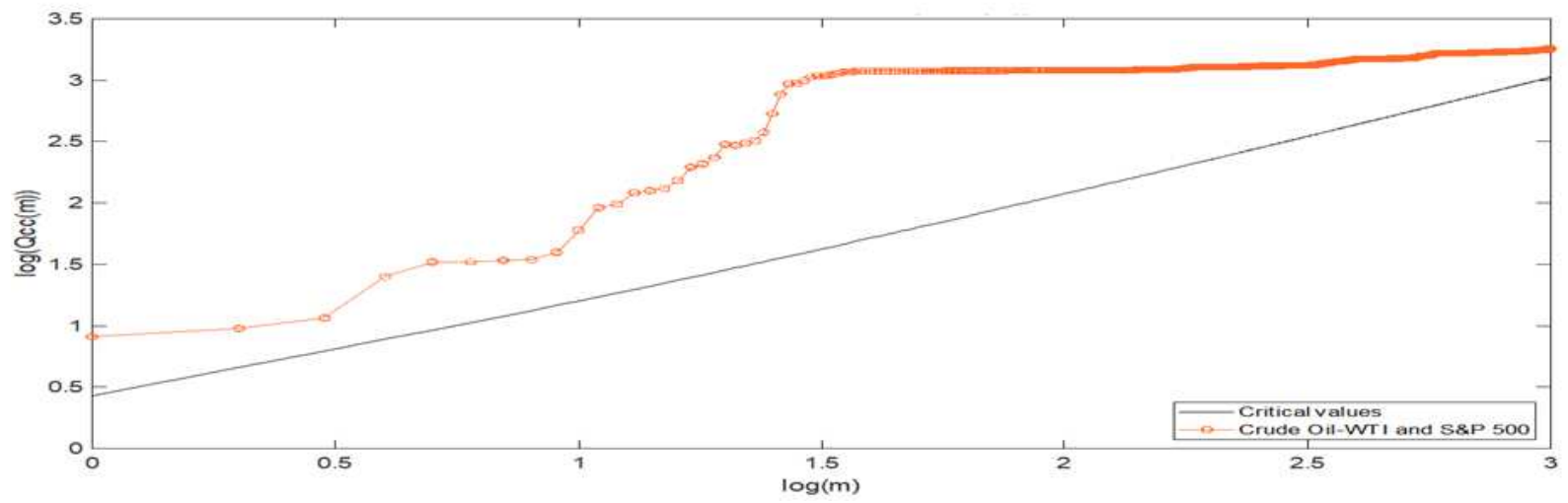

Figure 2: $\log -\log$ cross-correlation test $Q_{c c}(m)$ for full sample period 
Notes: the plot represents the Log-Log cross-correlation statistics $Q_{c c}(m)$ for two time series pairs, full sample. The test statistic $Q_{c c}(m)$ is approximately $\chi^{2}(m)$ distributed with $m$ degrees of freedom. The critical value of the cross-correlation statistics $\chi^{2}(m)$ is represented by the black line with at 5\% significant level. The red line denotes the cross-correlation statistic $Q_{c c}(m)$.

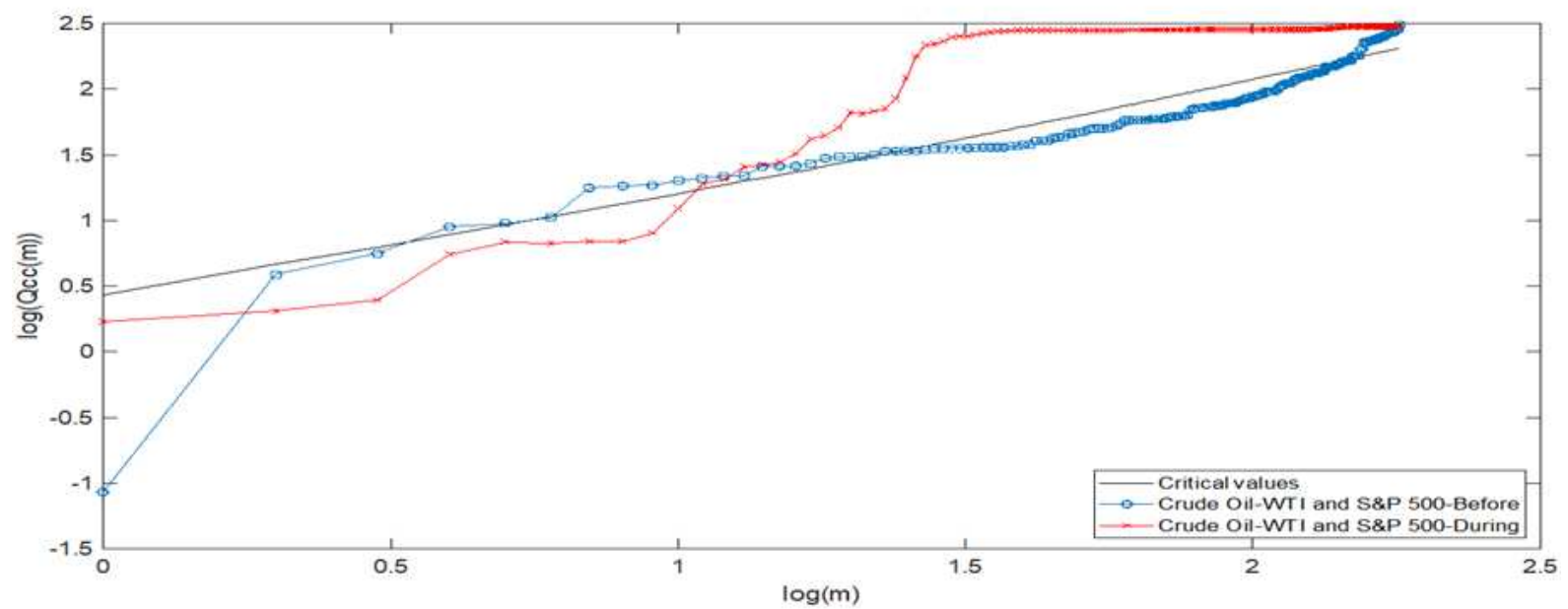

Figure 3: $\log -\mathrm{Log}$ cross-correlation test $Q_{c c}(m)$ for two periods

Notes: the plot represents the Log-Log cross-correlation statistics $Q_{c c}(m)$ for two time series pairs, full sample. The test statistic $Q_{c c}(m)$ is approximately $\chi^{2}(m)$ distributed with $m$ degrees of freedom. The critical value of the cross-correlation statistics $\chi^{2}(m)$ is represented by the black line with at 5\% significant level. The red line denotes the cross-correlation statistic $Q_{c c}(m)$.

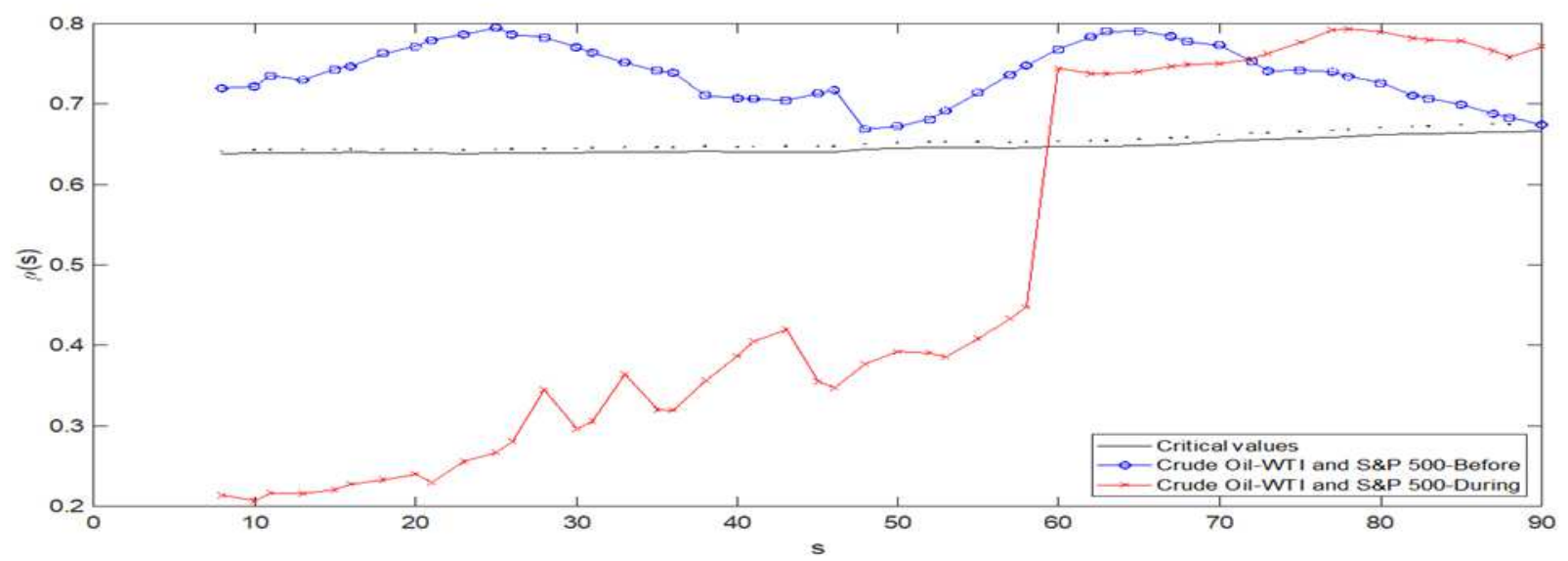

Figure 4: Cross-correlation coefficient $\rho_{D C C A}$ for two periods

Notes: the plot represents the detrended cross-correlation coefficient, $\rho(s)$, for different scale size s, varied from 10 to 90 . The black line is the critical value of $\chi^{2}$ and dot line is its 5\% critical values to test hypotheses $H 0: \rho(s)=0$ and H1: $\rho(s) \neq 0$,. The value of $\rho(s)$ ranges from -1 to 1 . A closer value to 1 means the series pair has a higher cross- correlation. When $\rho(s)=0$, there exists no cross-correlation. 

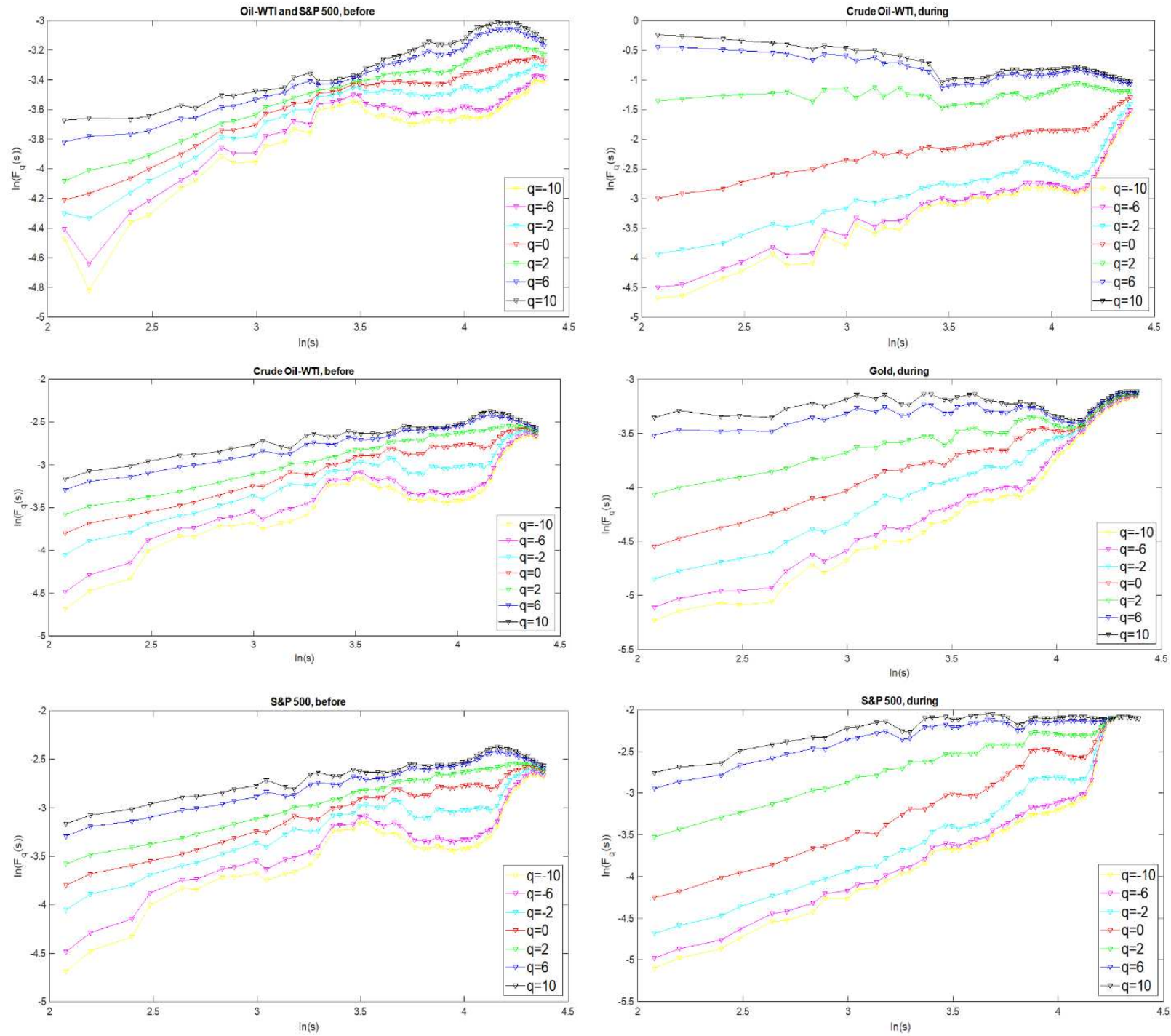

Figure 5: $\log$-log fluctuation function versus time scale Notes: the plots represent the $\log -\log$ of fluctuation functions $F_{q}(s)$ versus fractal order q values $(\mathrm{q}=-10,-8, \ldots, 8,10)$, based on a varied $\mathrm{s}$. 


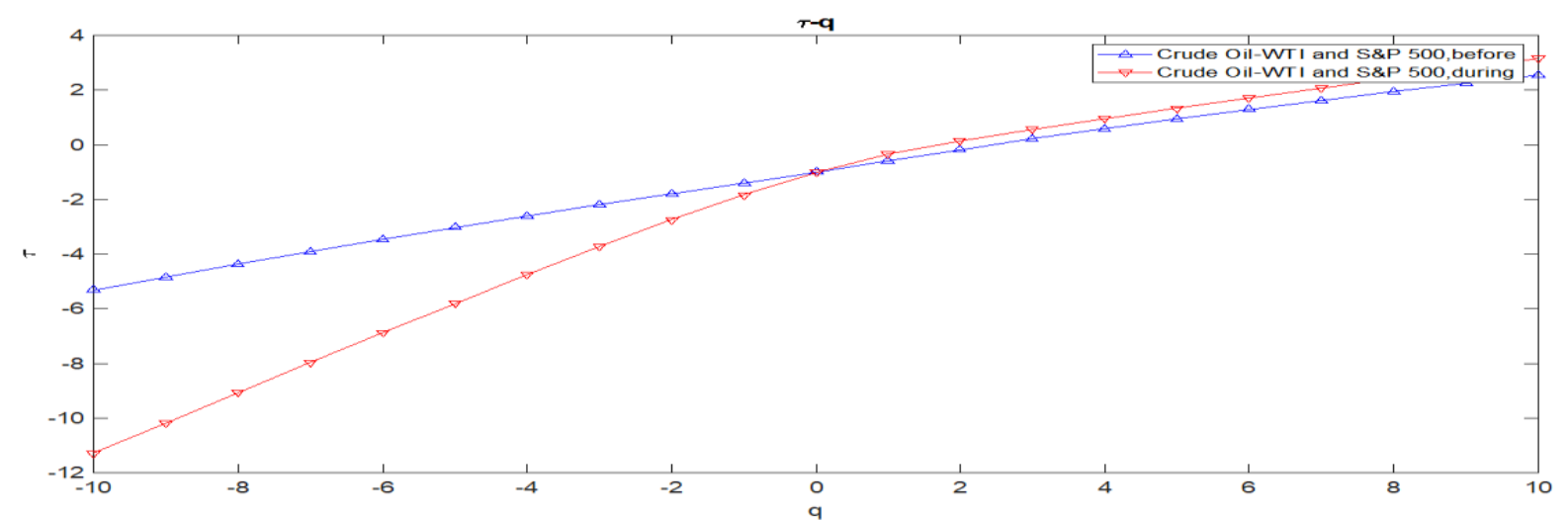

Figure 6: Cross correlation scaling exponent $\tau(q) \sim q$

Notes: the plot represents the cross correlation scaling exponents between oil and US equities with q varying from -10 to 10 .
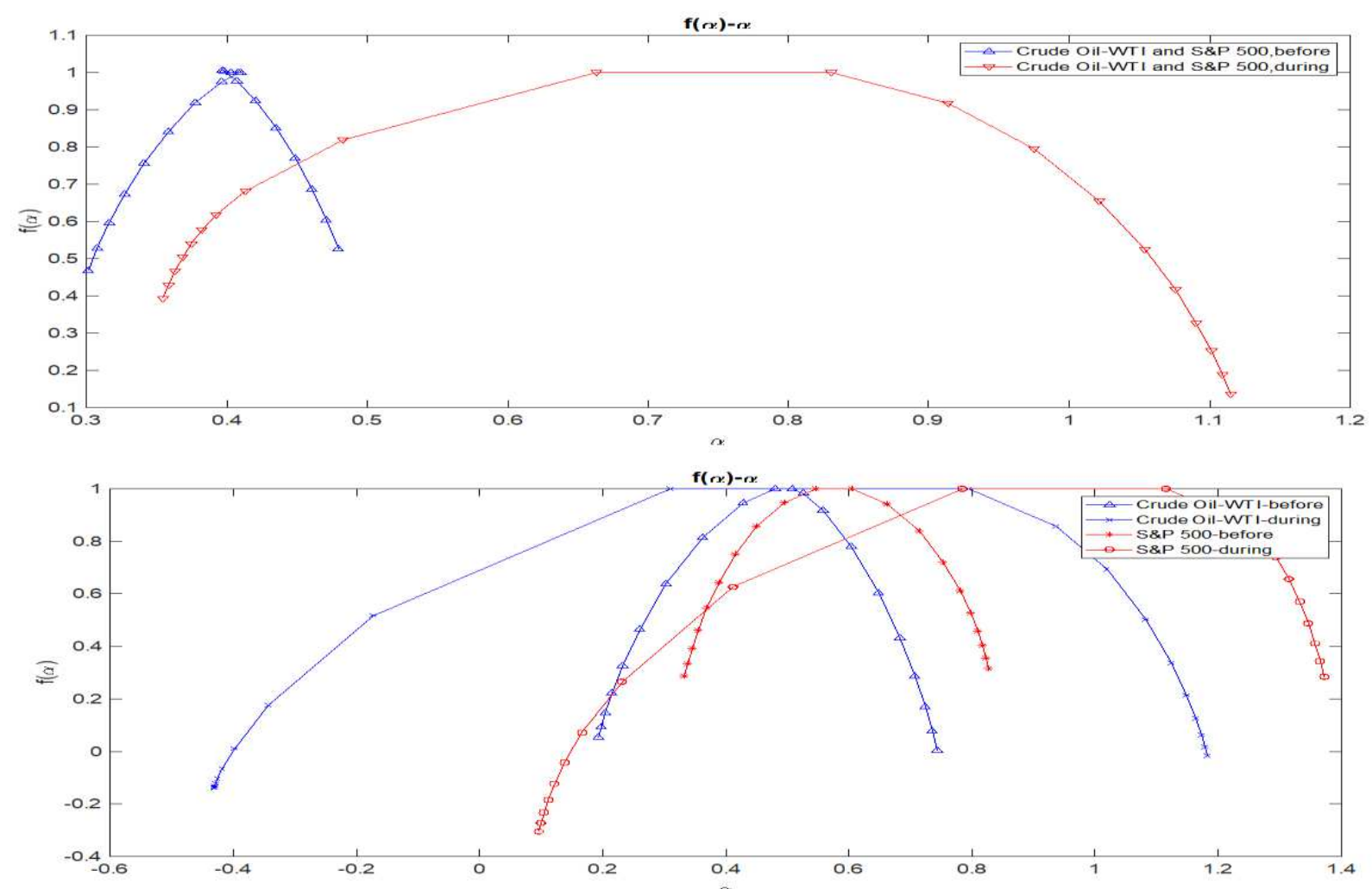

Figure 7: The multifractal spectrums $f(\alpha)$ of cross-correlations

Notes: the plots represent the multifractal spectrums $f(\alpha)$ of cross-correlations. The largest spectrum indicates strong multifractality and smallest spectrum indicates the weak multifractal. 

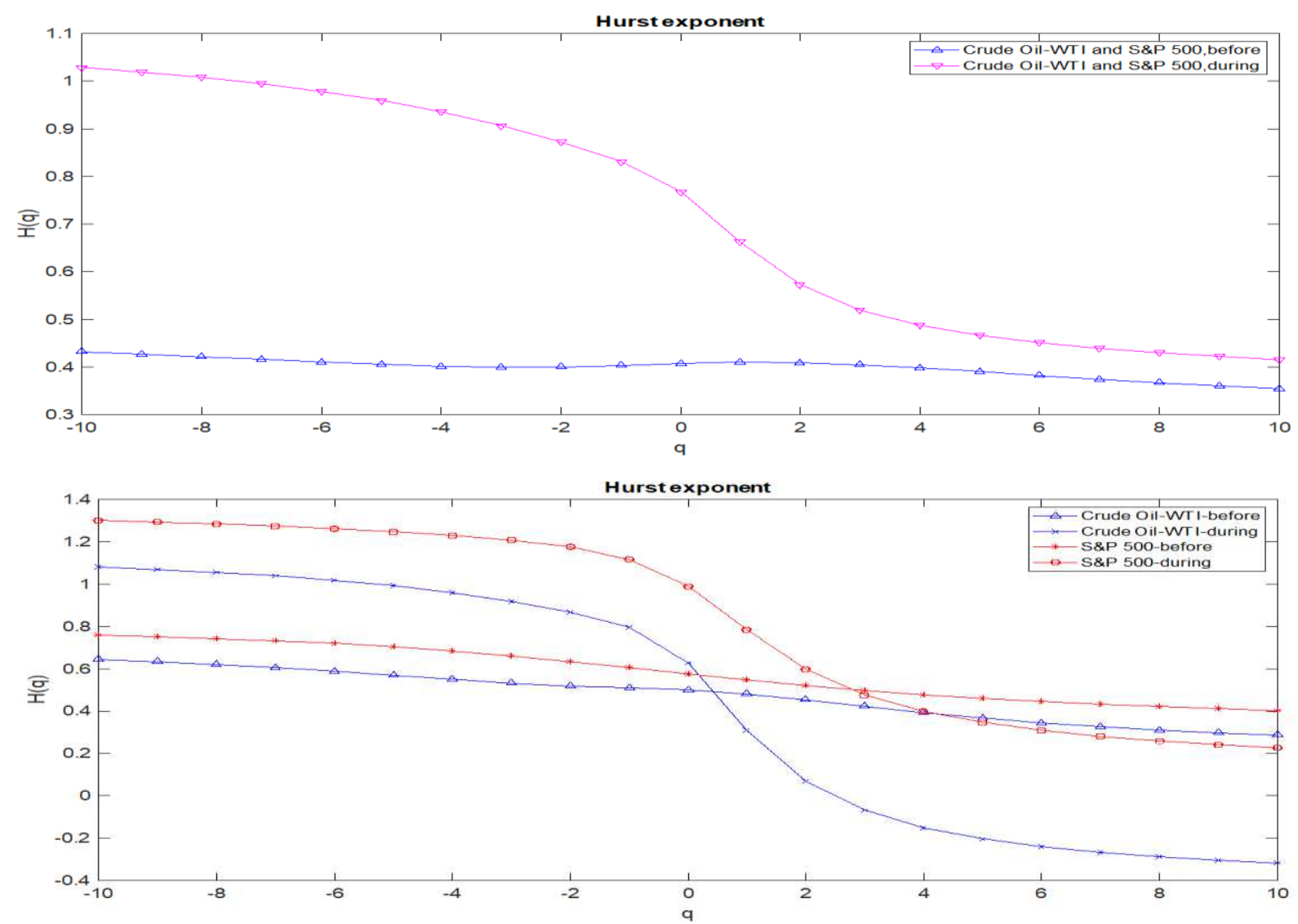

Figure 8: cross- correlation generalized Hurst exponents

Notes: the plots represent the Generalized Hurst exponents $h(q)$ spectrum of cross-correlations with q ranges from -10 to 10 . The horizontal axis is represented the singularity strength $h(q)$ and the vertical axis is represented the singularity spectrum q.
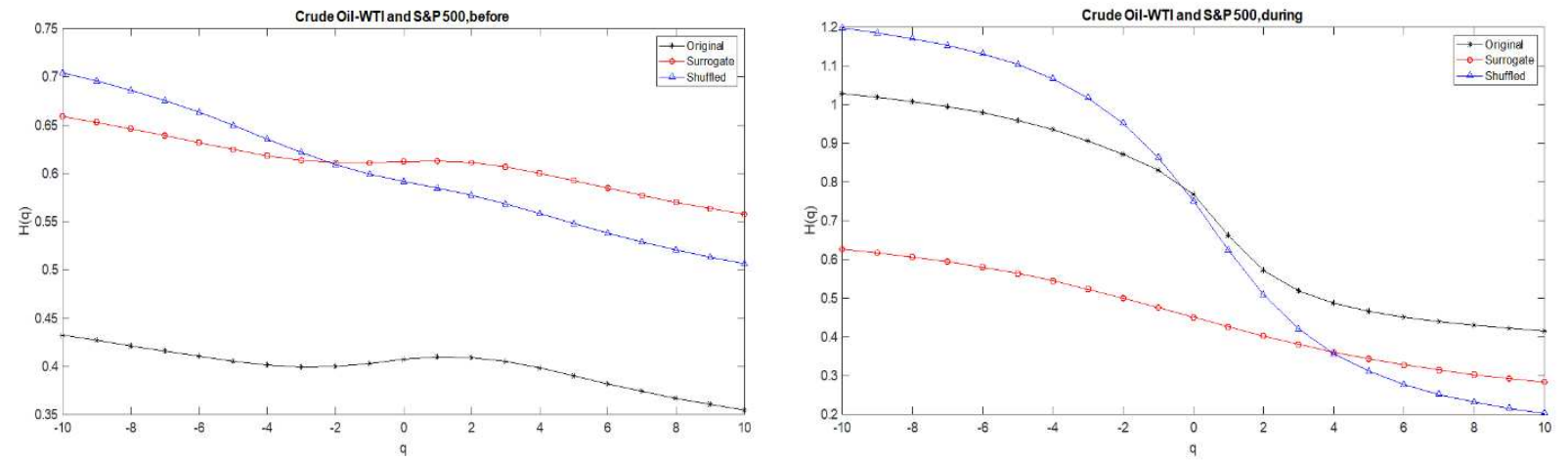

Figure 9: Scaling exponents of original, shuffled, and surrogated series time series

Notes: the plots represent the Generalized Hurst exponent $\mathrm{h}(\mathrm{q})$ of the original, shuffled and surrogated series time series with $\mathrm{q}$ ranges from -10 to 10 . The horizontal axis is represented the singularity strength $\mathrm{h}(\mathrm{q})$ and the vertical axis is represented the singularity spectrum $\mathrm{q}$. 

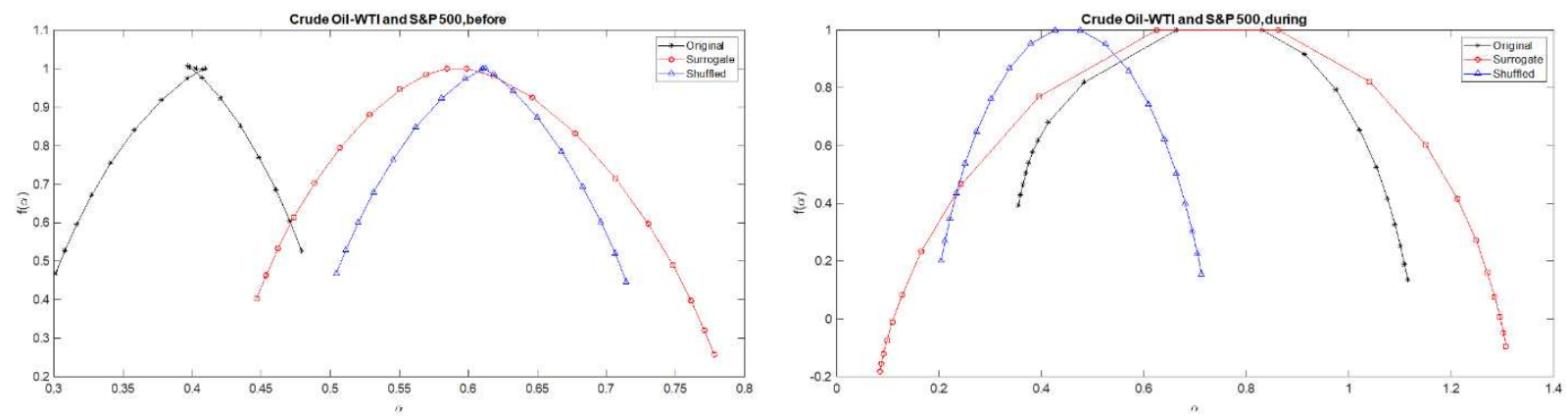

Figure 10: Multifractal spectrums of original, shuffled, and surrogated series time series

Notes: the plots represent the multifractal spectrum $f(\alpha)$ obtained by a Legendre transformation of $\tau(q)$, as a function of the singularity strength $\alpha$ for the original, shuffled, and surrogated series time series.

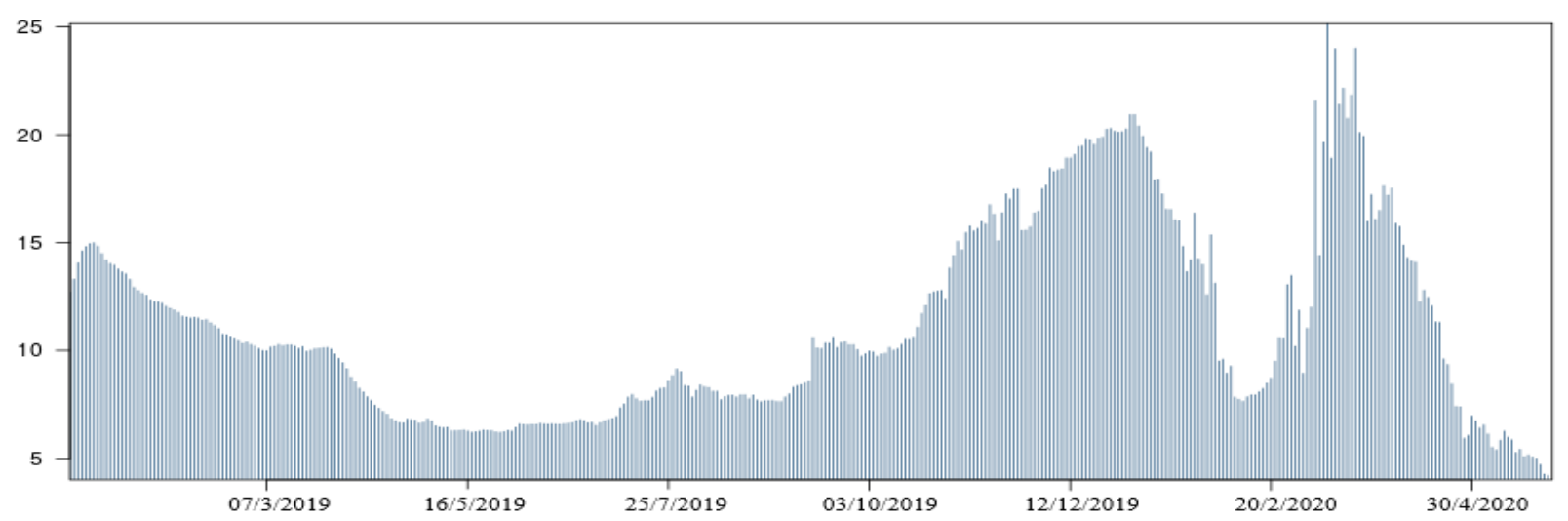

Figure 11: Total connectedness index (TCI)

Notes: The plot displays the dynamic total connectedness index (TCI) based on TVP-VAR with the lag length of order 2 and a 12 -step-ahead forecast. 
(a) Crude Oil-WTI TO S\&P 500

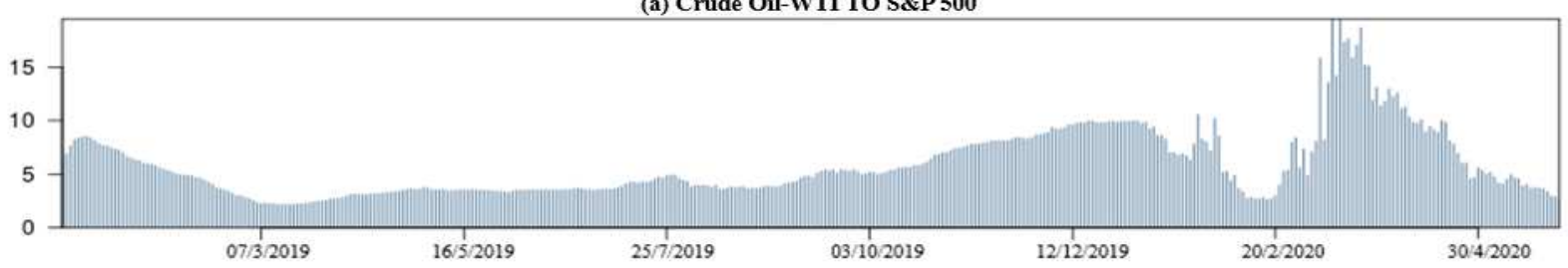

(b) S\&P 500 TO Crude Oil-WTI

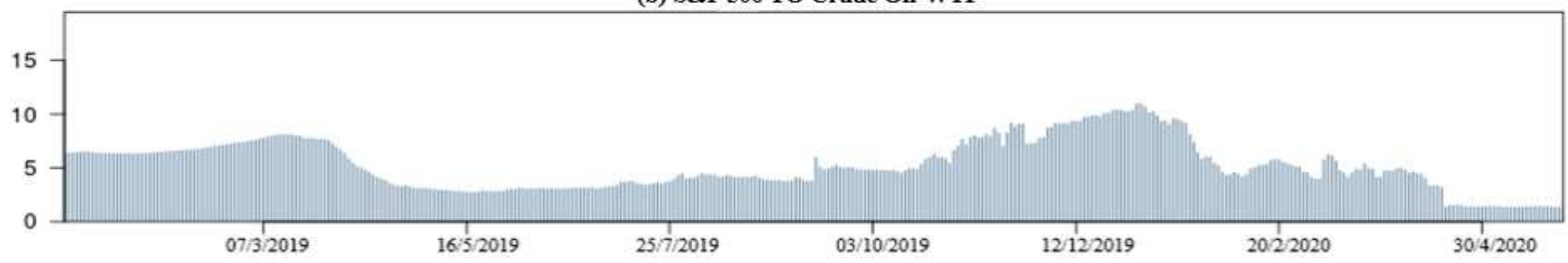

Figure 12: Total directional connectedness TO other

Note: Results are based on a TVP-VAR model with lag length of order 2 (BIC) and a 12-step-ahead forecast.

(a) NET Crude Oil-wT

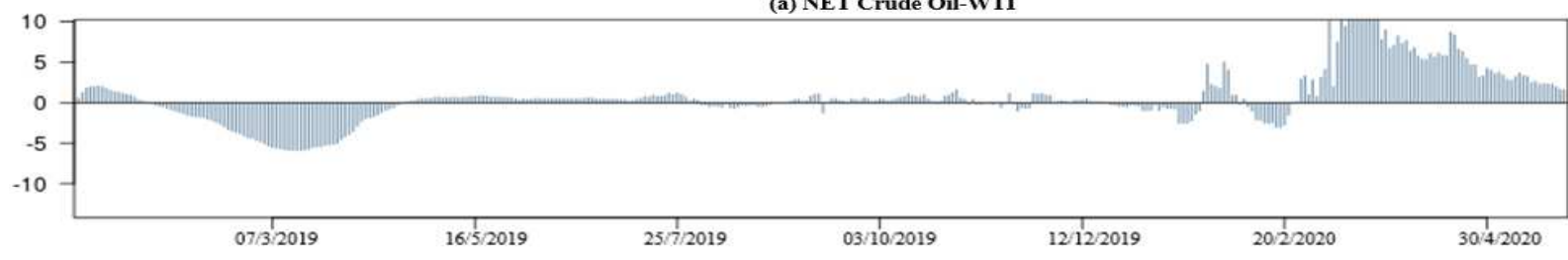

(b) NET S\&P 500

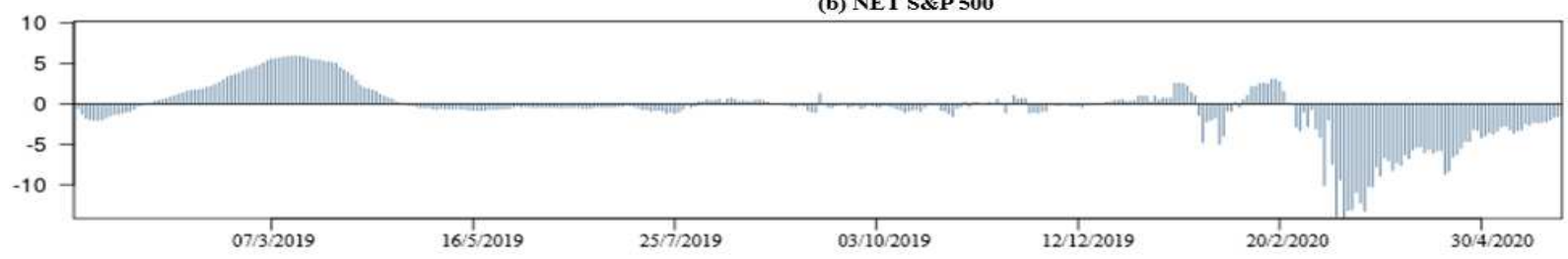

Figure 13: Net total directional connectedness

Note: Results are based on a TVP-VAR model with lag length of order 2 (BIC) and a 12-step-ahead forecast. Positive (negative) values indicate that $i$ is a net transmitter (receiver) of shocks to the $j$. 

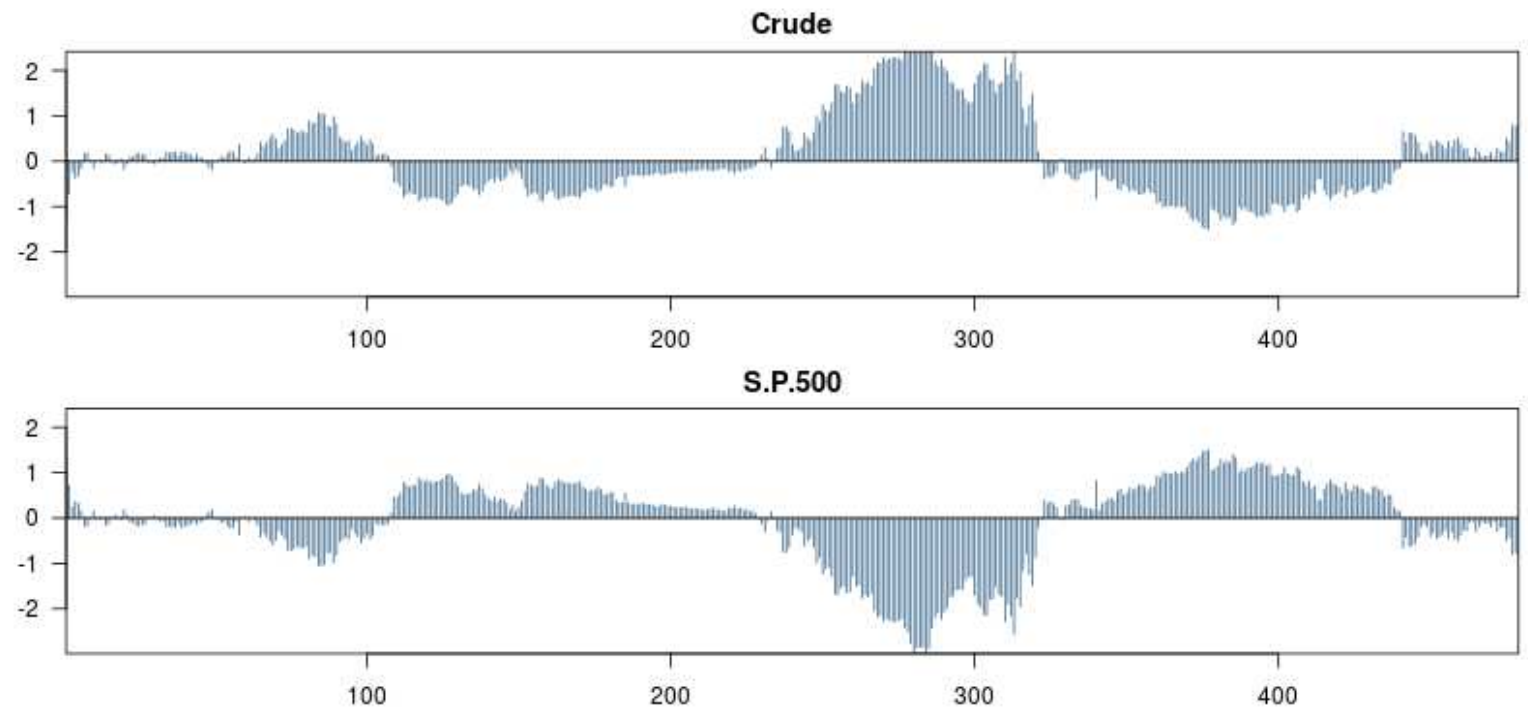
Figures

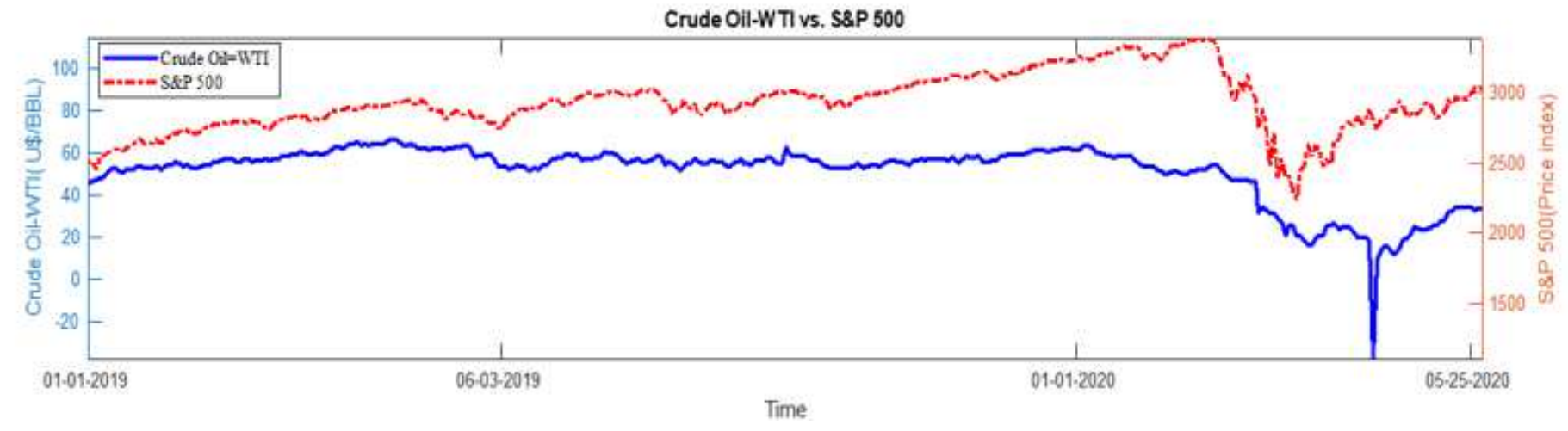

\section{Figure 1}

Time evolutions for the time series

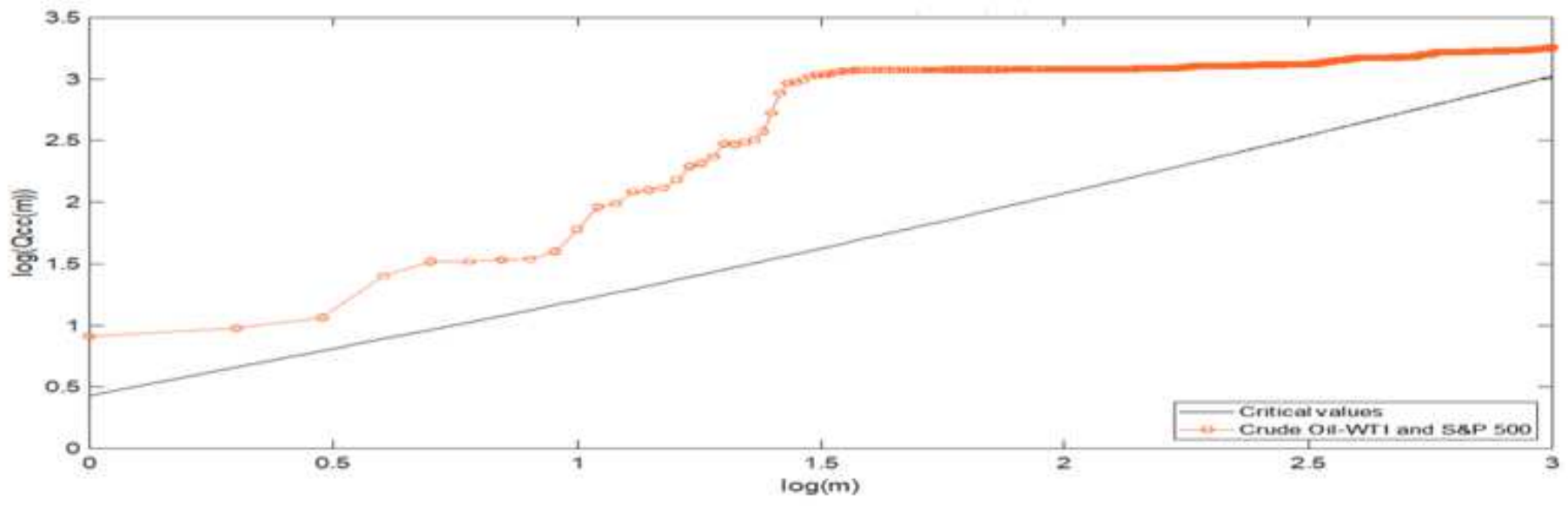

Figure 2

See manuscript for full figure caption. 


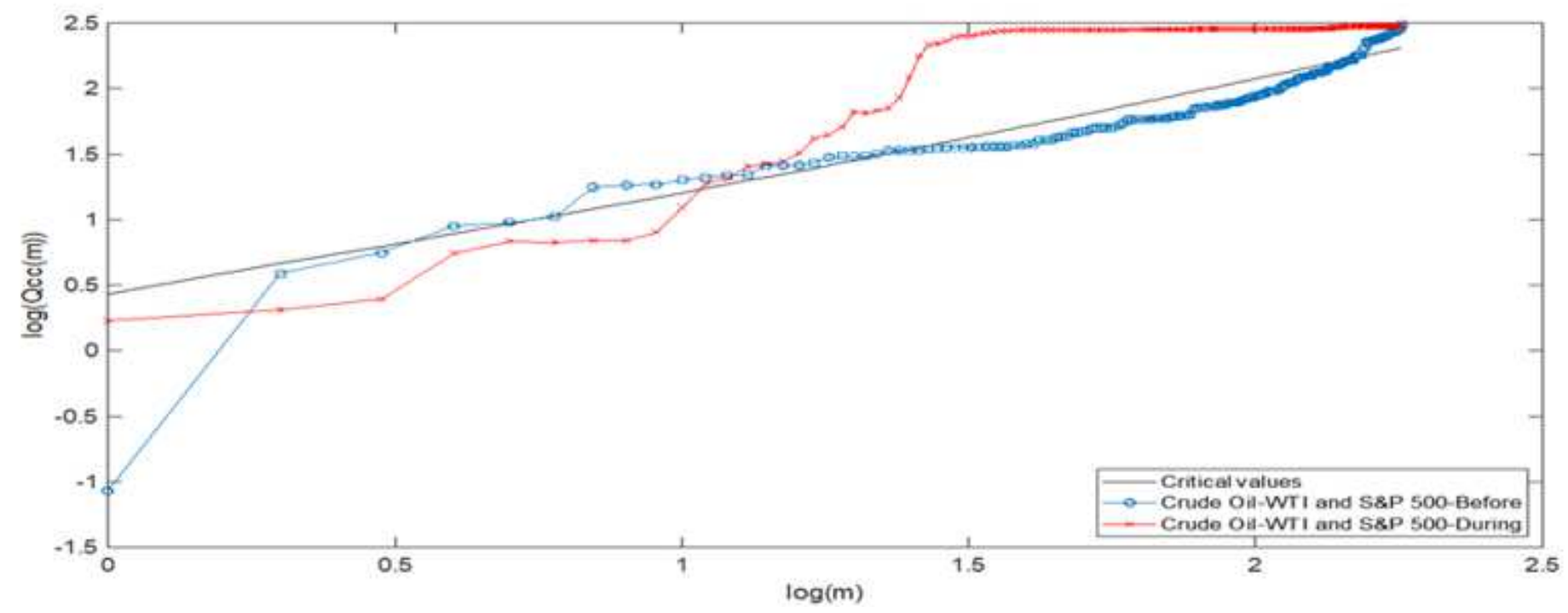

Figure 3

See manuscript for full figure caption.

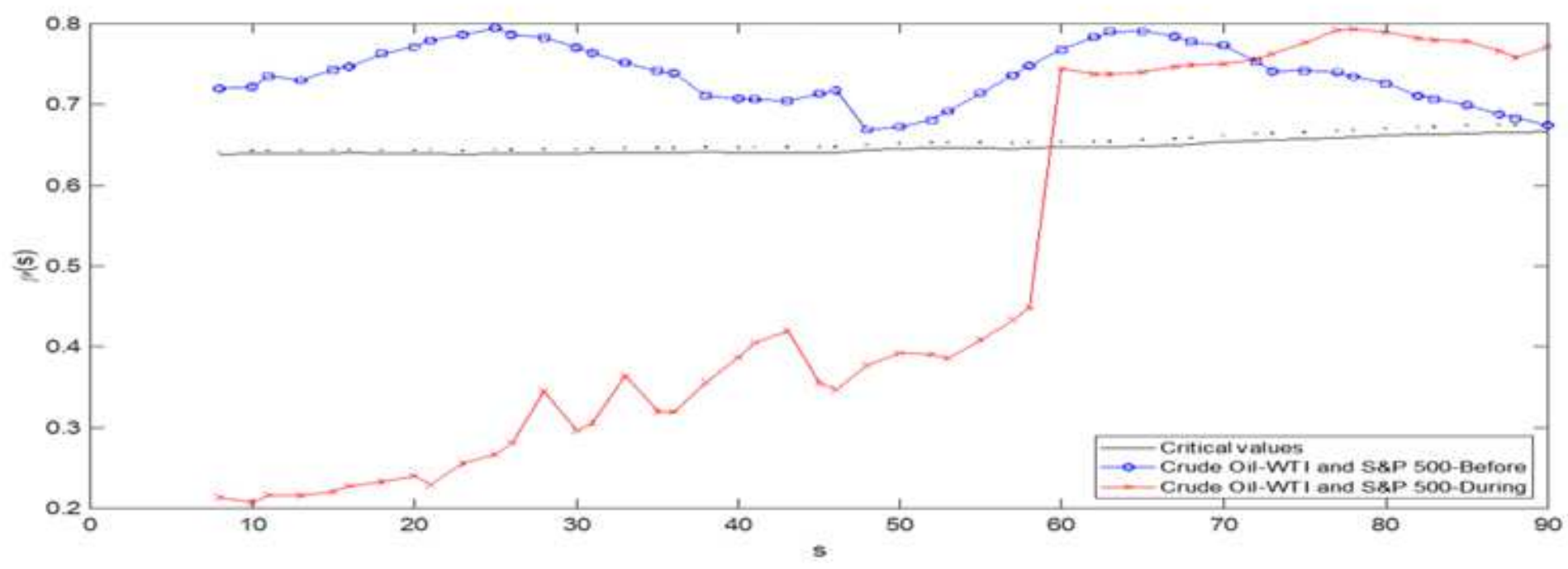

Figure 4

See manuscript for full figure caption. 

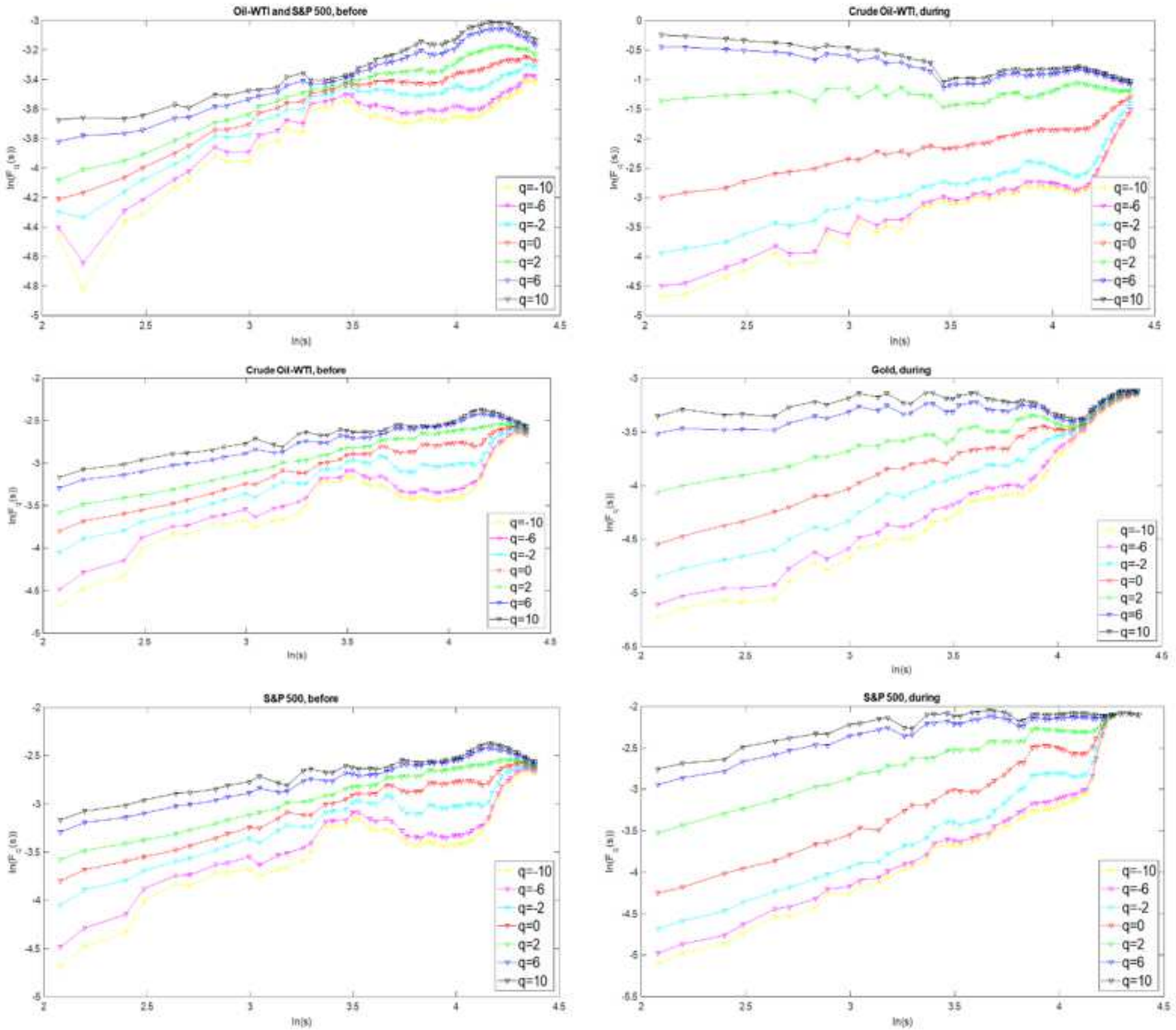

Figure 5

See manuscript for full figure caption. 


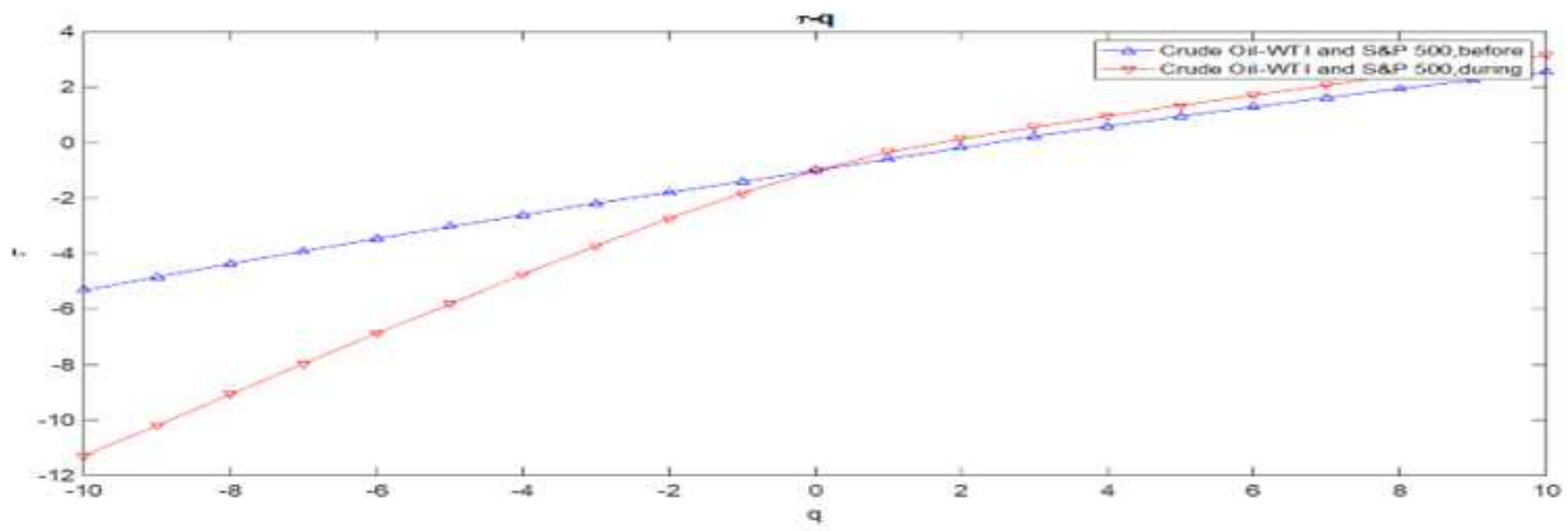

Figure 6

Cross correlation scaling exponent $\mathrm{t}(\mathrm{q}) \sim \mathrm{q}$ Notes: the plot represents the cross correlation scaling exponents between oil and US equities with q varying from -10 to 10 .
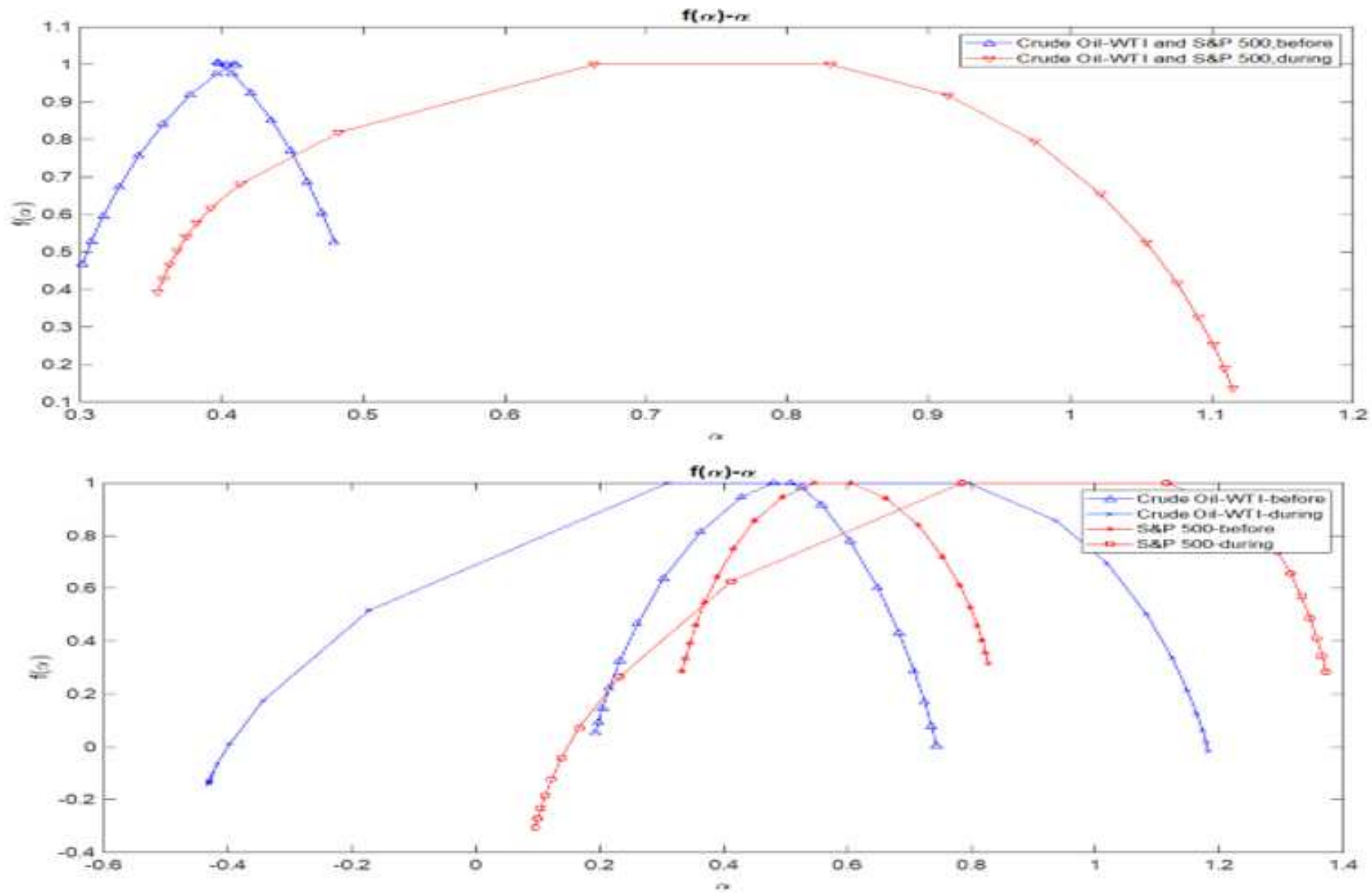

\section{Figure 7}

The multifractal spectrums $f(a)$ of cross-correlations Notes: the plots represent the multifractal spectrums $f(a)$ of cross-correlations. The largest spectrum indicates strong multifractality and smallest spectrum 
indicates the weak multifractal.
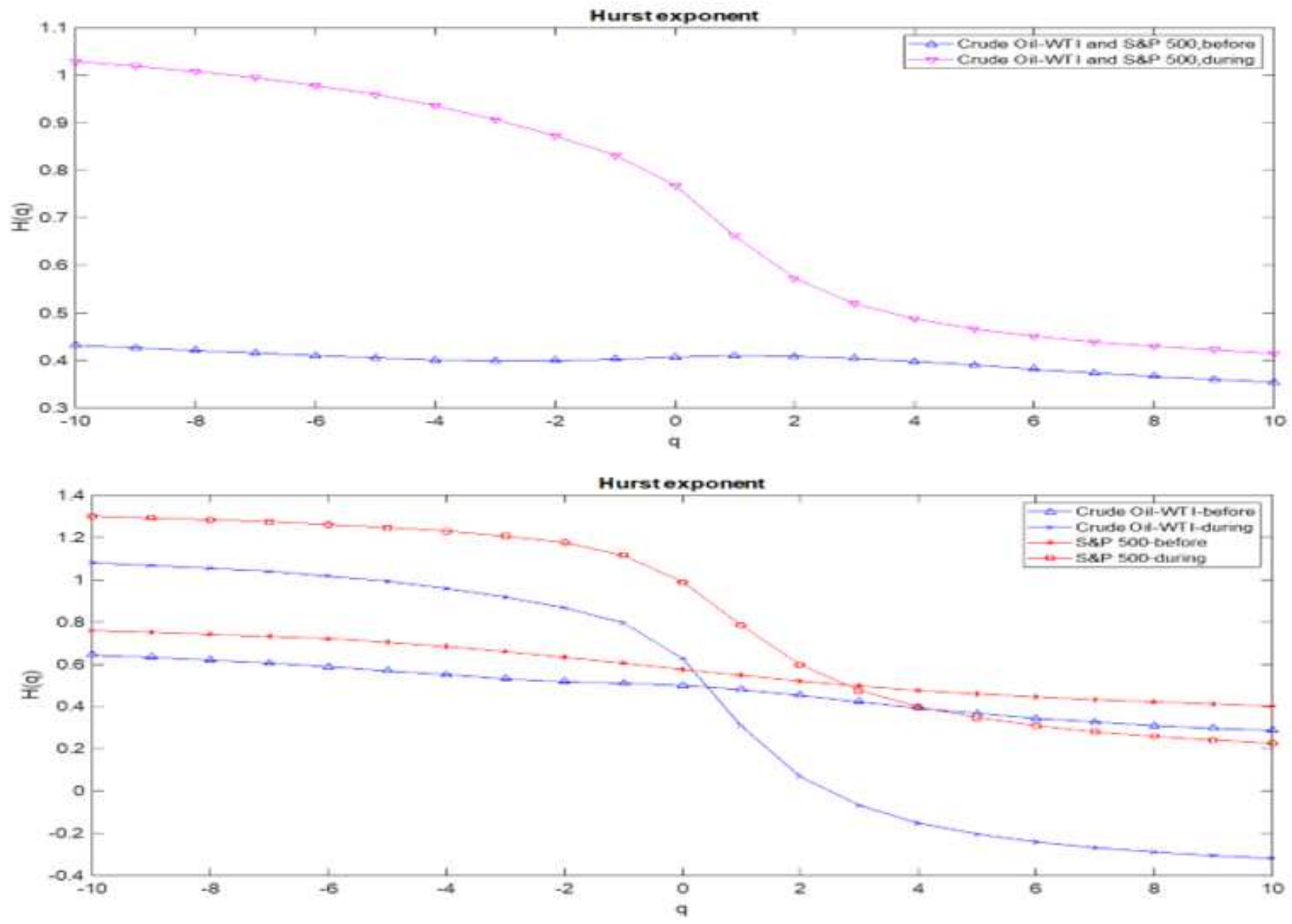

\section{Figure 8}

cross- correlation generalized Hurst exponents Notes: the plots represent the Generalized Hurst exponents $h(q)$ spectrum of cross-correlations with q ranges from -10 to 10 . The horizontal axis is represented the singularity strength $\mathrm{h}(\mathrm{q})$ and the vertical axis is represented the singularity spectrum $\mathrm{q}$.
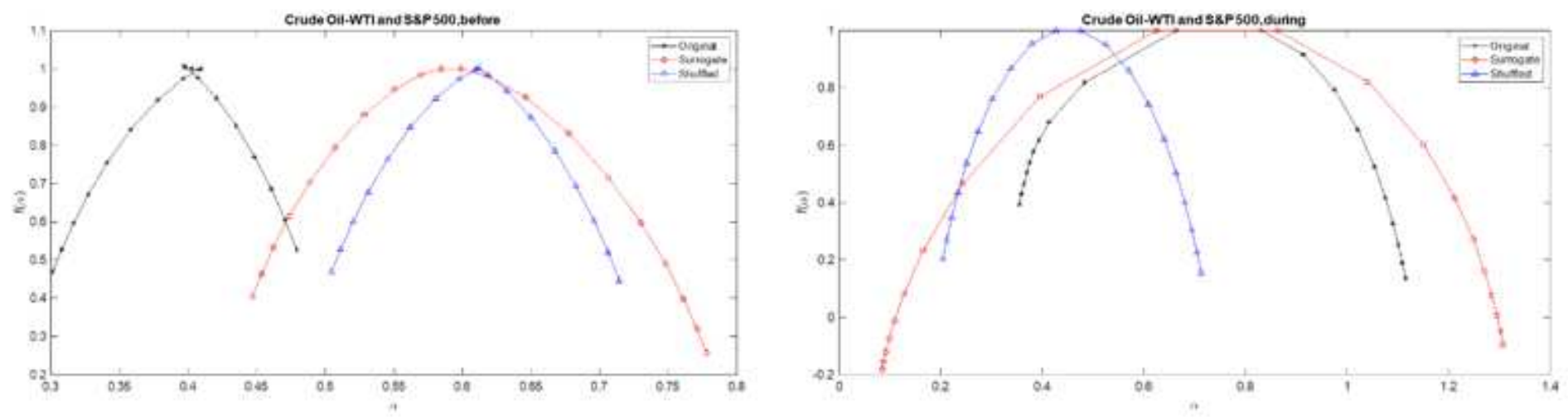

Figure 9 
Scaling exponents of original, shuffled, and surrogated series time series Notes: the plots represent the Generalized Hurst exponent $\mathrm{h}(\mathrm{q})$ of the original, shuffled and surrogated series time series with q ranges from -10 to 10 . The horizontal axis is represented the singularity strength $h(q)$ and the vertical axis is represented the singularity spectrum q.

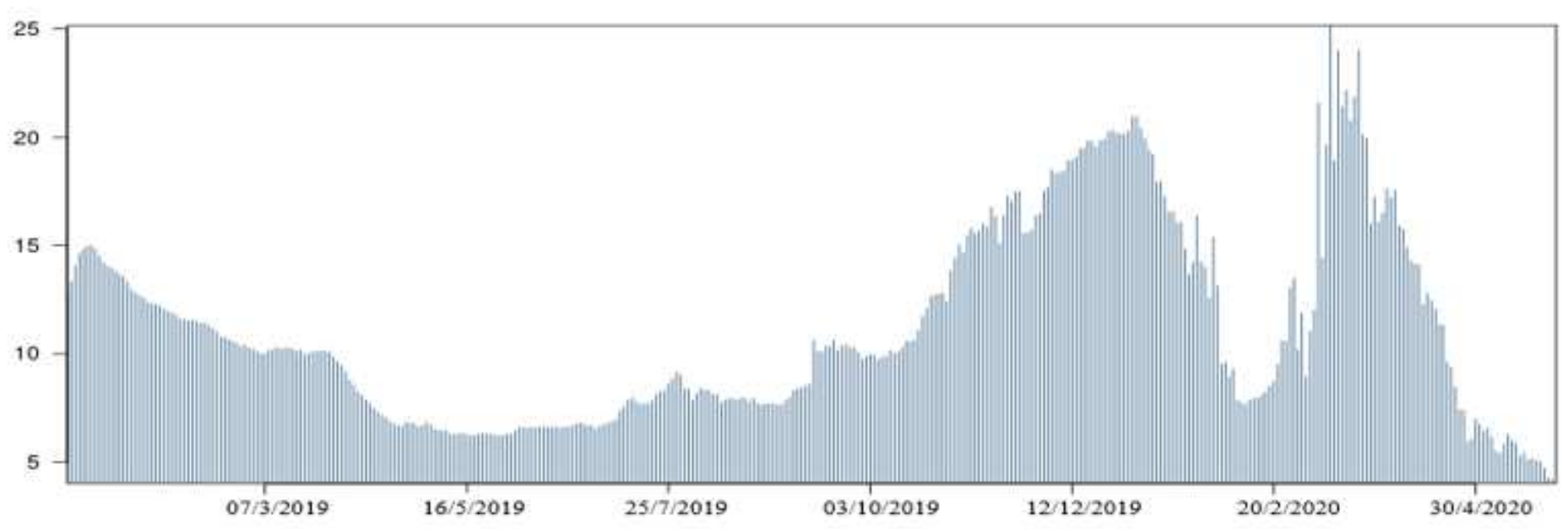

Figure 10

Multifractal spectrums of original, shuffled, and surrogated series time series Notes: the plots represent the multifractal spectrum $f(a)$ obtained by a Legendre transformation of $\tau(q)$, as a function of the singularity strength a for the original, shuffled, and surrogated series time series.

(a) Crude Oil-WTI To S\&P 500

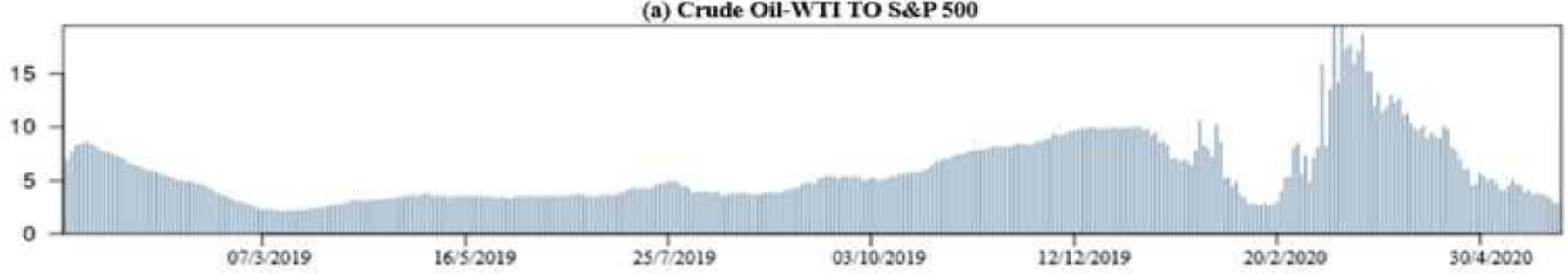

(b) S\&P 500 TO Crude Oil-WTI

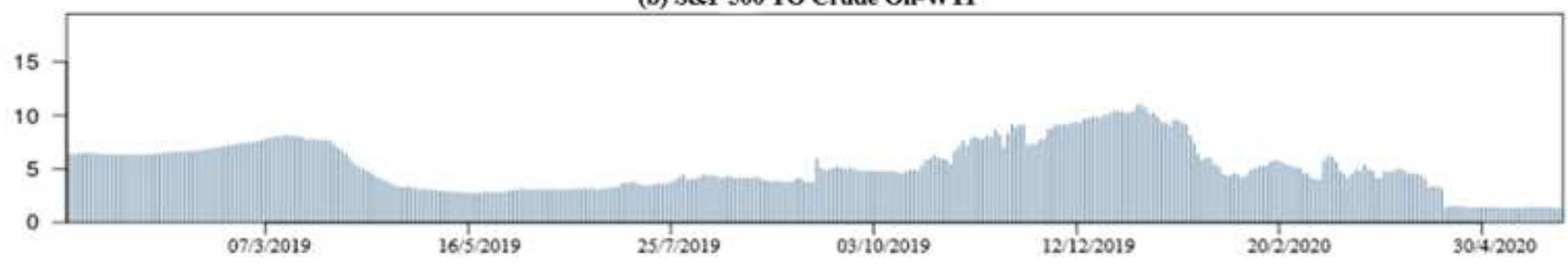

\section{Figure 11}

Total connectedness index ( $\mathrm{TCl}$ ) Notes: The plot displays the dynamic total connectedness index (TCl) based on TVP-VAR with the lag length of order 2 and a 12-step-ahead forecast. 
(a) NET Crude Oil-WT

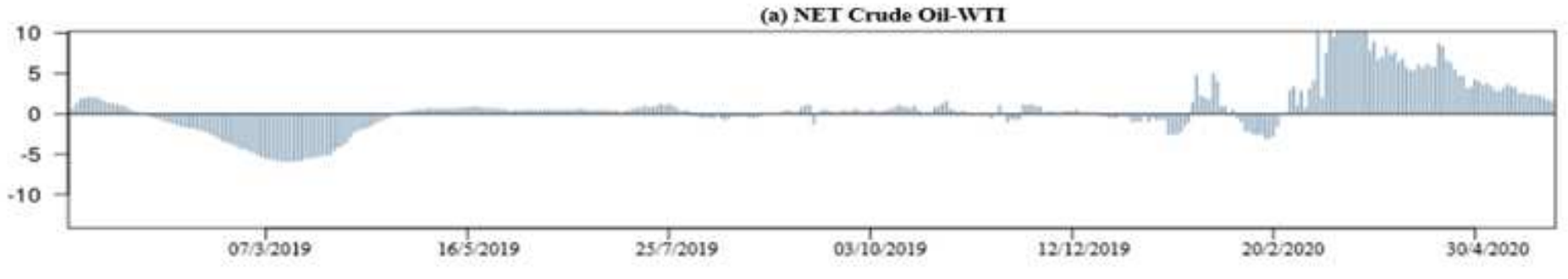

(b) NET S\&P 500

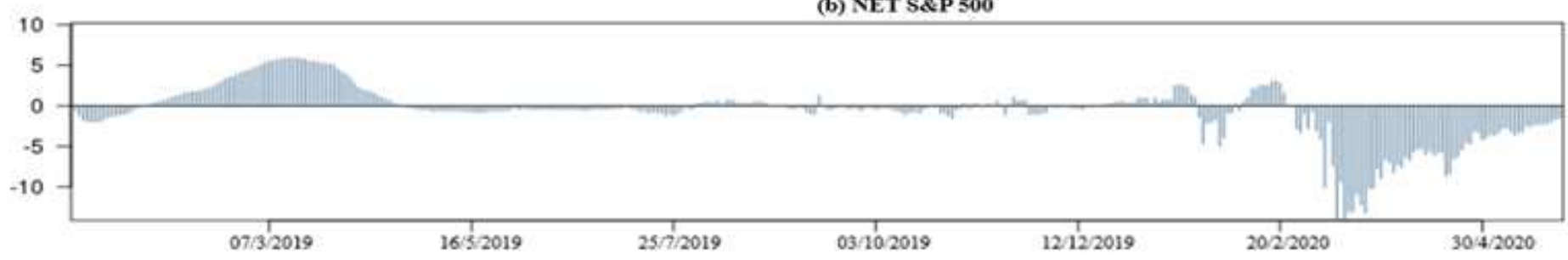

Figure 12

Total directional connectedness TO other Note: Results are based on a TVP-VAR model with lag length of order 2 (BIC) and a 12-step-ahead forecast.
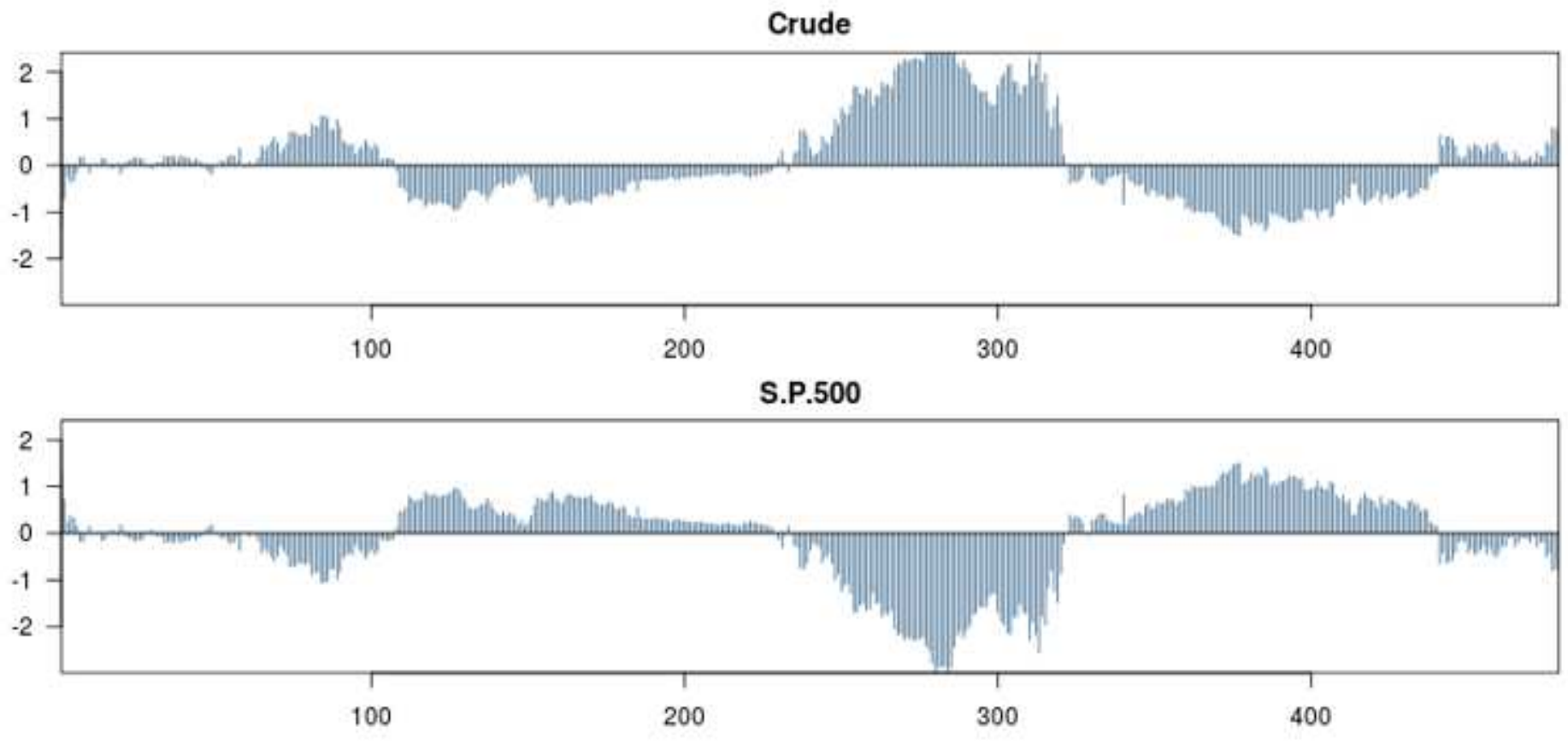

\section{Figure 13}

Net total directional connectedness Note: Results are based on a TVP-VAR model with lag length of order 2 (BIC) and a 12-step-ahead forecast. Positive (negative) values indicate that $\mathrm{i}$ is a net transmitter (receiver) of shocks to the $\mathrm{j}$.

\section{Supplementary Files}


This is a list of supplementary files associated with this preprint. Click to download.

- Dataspreadsheet.xlsx 